\title{
Evidence of discrete energy states and cluster-glass behavior in $\mathrm{Sr}_{2-x} \mathrm{La}_{x} \mathrm{CoNbO}_{6}$
}

\author{
Ajay Kumar $\odot,{ }^{1}$ B. Schwarz $\odot,{ }^{2}$ H. Ehrenberg $\odot,{ }^{2}$ and R. S. Dhaka $\odot^{1, *}$ \\ ${ }^{1}$ Department of Physics, Indian Institute of Technology Delhi, Hauz Khas, New Delhi, 110016, India \\ ${ }^{2}$ Institute for Applied Materials (IAM), Karlsruhe Institute of Technology (KIT), 76344, Eggenstein-Leopoldshafen, Germany
}

(Received 11 June 2020; revised 11 August 2020; accepted 14 October 2020; published 12 November 2020)

We report the detailed analysis of specific heat $\left[C_{P}(T)\right]$ and ac susceptibility for magnetically frustrated $\mathrm{Sr}_{2-x} \mathrm{La}_{x} \mathrm{CoNbO}_{6}(x=0-1)$ double perovskites to understand low-temperature complex magnetic interactions and their evolution with $x$. Interestingly, the observed Schottky anomaly in the $x \leqslant 0.4$ samples shifts gradually toward higher temperatures with magnetic field as well as $x$, and the analysis reveals the persistence of the discrete energy states in these samples resulting from the spin-orbit coupling and octahedral distortion. Moreover, the extracted values of the Landé $g$ factor indicate the existence of high-spin state $\mathrm{Co}^{3+}$ ions energetically close to a nonmagnetic low-spin state. The specific-heat data show the $\lambda$-type anomaly for the $x \geqslant 0.6$ samples due to evolution of the long-range antiferromagnetic ordering. Our analysis of low-temperature $C_{P}(T)$ data for the $x \geqslant 0.6$ samples demonstrates the 3D isotropic Heisenberg antiferromagnetic (AFM) interactions and the temperature-induced second-order AFM-paramagnetic phase transition. More interestingly, we demonstrate the presence of the free $\mathrm{Co}^{2+}$-like Kramers doublet ground state in the $x=1$ sample. Further, the ac susceptibility and time evolution of the magnetization data reveal the low-temperature cluster-glass-like behavior in the $x=$ $0-0.4$ samples, where spin-spin correlation strength decreases with $x$.

DOI: 10.1103/PhysRevB.102.184414

\section{INTRODUCTION}

Frustrated antiferromagnetic (AFM) insulators have attracted great interest due to their peculiar magnetic ground states, namely, spin glass (SG), cluster glass, spin liquid, spin ice, etc., unlike conventional long-range antiferromagnets [1-4]. In this context, double perovskite oxides having general formula $A_{2} B B^{\prime} \mathrm{O}_{6}$ ( $A$ : rare-earth/alkali-earth metals, $B / B^{\prime}$ : transition metals) further incorporate the rich physics because of their well-established stable structure and coordination environment, which gives flexibility to accommodate the wide range of cations at $A$ and/or $B / B^{\prime}$ site(s), resulting in the interesting physical properties like low-field colossal magnetoresistance, half-metallicity, superconductivity, and geometrical as well as magnetic frustration, etc. [5-10]. The large ionic and valence mismatch between $B$-site cations are known to favor the alternating ordering of corner-shared $B_{0}$ and $B^{\prime} \mathrm{O}_{6}$ octahedra in these compounds, forming the threedimensional rock-salt-like ordered structure $[11,12]$. An ideal cubic double-perovskite structure can be visualized as the two interpenetrating face-centered cubic sublattices, which give rise to frustration between the spins, if only one of the $B$-site cations is magnetic and antiferromagnetically coupled with its nearest neighbor, analogous to pyrochlore and kagomé lattices

\footnotetext{
*rsdhaka@physics.iitd.ac.in
}

Published by the American Physical Society under the terms of the Creative Commons Attribution 4.0 International license. Further distribution of this work must maintain attribution to the author(s) and the published article's title, journal citation, and DOI.
$[1,7]$. The short-range magnetic correlations are present in such compounds even well above the magnetic-transition temperature, accompanied by the lowering in crystal symmetry $[8,9]$.

In this direction, Co-based oxides have been extensively studied due to the various possible oxidation and spin states of $\mathrm{Co}$, which give rise to exotic magnetic ground states [13-15]. For example, a delicate competition between Hund's exchange energy and crystal field (CF) splitting of $\mathrm{Co}^{3+}$ ions $\left(3 d^{6}\right)$ in the case of $\mathrm{LaCoO}_{3}$ results in the considerably small energy difference between low spin (LS; $\mathrm{t}_{2 g}^{6} \mathrm{e}_{g}^{0}$ ) and high spin (HS; $\mathrm{t}_{2 g}^{4} \mathrm{e}_{g}^{2}$ ) states [16-18]. The perturbation by the chemical substitution at $\mathrm{La}$ and/or Co site(s) can sensitively alter their relative population and hence the wide range of physical properties ranging from ferromagnetic metals to $\mathrm{SG}$ insulators [19-22]. It is important to note here that the strong CF in the case of $\mathrm{Co}^{3+}$ in the octahedral coordination is expected to lift the orbital degeneracy and hence quench the orbital angular momentum, as the band-structure calculations suggest the presence of the continuous energy band of $3 d^{6}$ states $[23,24]$. However, electron spin resonance measurements by Noguchi et al. suggest the persistence of the discrete energy levels in $\mathrm{LaCoO}_{3}$ by claiming the existence of the HS spin-orbit triplet above the nonmagnetic LS ground state [25]. This was further supported by the theoretical work using the CF splitting, spinorbit coupling, octahedral distortion and Zeeman's splitting in the Hamiltonian, where the $\mathrm{CF}$ was considered in the weak limit as compared to the electron-electron interactions within a $3 d$ shell, i.e., starting from the free-ion-like ${ }^{5} \mathrm{D}$ ground term [26]. In the same line, it is interesting that the presence of the low-temperature Schottky anomaly in the specific-heat data of $\mathrm{LaCoO}_{3}$ indicates around $0.5 \mathrm{meV}$ energy splitting 
between the trigonal CF-split singlet and a doublet of HS spin-orbit triplet with $g$ factor $\sim 3.5$, where the magnetic field lifts the degeneracy of the excited doublet [27]. These results are consistent with the inelastic neutron scattering study by Podlesnyak et al. [28] and suggest the presence of the nonnegligible orbital magnetic moment in the compound.

Also, it is important to note that $\mathrm{Co}^{2+}\left(3 d^{7}\right)$ in the octahedral coordination is more likely to preserve the free-ion-like energy levels due to weaker $\mathrm{CF}$ as compared to $\mathrm{Co}^{3+}$, resulting in the most stable HS state $\left(t_{2 g}^{5} e_{g}^{2}\right)$ with a significantly large unquenched orbital magnetic moment due to the triply degenerate ${ }^{4} T_{1 g}$ ground term [29-31]. Therefore, it is vital to investigate the possibility of the free-ion-like energy scheme in the compounds with mixed $\mathrm{Co}^{2+}$ and $\mathrm{Co}^{3+}$ ions having the significant spin frustration, as $\mathrm{HS} \mathrm{Co}^{2+}$ favors AFM exchange interactions [30,31]. For example, recently, we investigated SG behavior in Nb-substituted $\mathrm{LaCoO}_{3}$, where each $\mathrm{Nb}^{5+}$ transforms two $\mathrm{Co}^{3+}$ ions into $\mathrm{Co}^{2+}$ [21]. However, in the case of double perovskites, additional $B-\mathrm{O}-B$ and $B^{\prime}-\mathrm{O}-B^{\prime}$ exchange interactions due to disorder at the $B$ site result in the magnetic frustration and hence suppresses the long-range magnetic ordering, which gives rise to the glassy magnetic ground states [32-34]. For example, a small energy difference between $\mathrm{Co}^{2+}-\mathrm{Mn}^{4+}$ and $\mathrm{Co}^{3+}-\mathrm{Mn}^{3+}$ states $(\approx 0.2 \mathrm{eV})$ gives rise to the mixed valence states of $\mathrm{Co}$ and $\mathrm{Mn}$ in $\mathrm{La}_{2} \mathrm{CoMnO}_{6}$, which results in cluster-glass-like behavior at low temperatures due to mixed FM and AFM interactions, owing to the antisite disorder $[32,35]$. However, despite the presence of predominantly $\mathrm{Co}^{2+}$ and $\mathrm{Mn}^{4+}$ ions in its analogous compound $\mathrm{EuMn}_{0.5} \mathrm{Co}_{0.5} \mathrm{O}_{3}$ as evident from the x-ray absorption spectroscopic (XAS) measurements, antisite disorder results in several competing exchange interactions and hence the SG ground state [36]. Recently, a SG-type ground state and exchange bias effect have been reported in disordered $\mathrm{La}_{2-x} \mathrm{Sr}_{x} \mathrm{CoFeO}_{6}$ compounds because multiple valence states of $\mathrm{Co}$ and $\mathrm{Fe}$ result in competing AFM-FM exchange interactions $[33,34]$. However, in these compounds, the presence of another magnetic $B$-site cation with the Co makes it difficult to understand the interesting exchange coupling between the multiple valence and spin states of individual Co ions.

In this regard, $\mathrm{Co}-\mathrm{Nb}$ systems are of particular interest as $\mathrm{Co}^{3+}-\mathrm{Nb}^{5+}$ lies at the border line of the phase diagram of ordered-disordered structures due to their moderate ionic and valence mismatch $(\Delta V=2, \Delta r \approx 0.06 \AA)$, whereas $\mathrm{Co}^{2+}-\mathrm{Nb}^{5+}$ lies largely toward the ordered structure $(\Delta V=$ $3, \Delta r \approx 0.06 \AA$ ) $[12,37]$. Azcondo et al. investigated the complex magnetic behavior and ground state of $\mathrm{Sr}_{2} \mathrm{CoNb}_{1-x} \mathrm{Ti}_{x} \mathrm{O}_{6}$ $(0 \leqslant x \leqslant 0.5)$, which show the low-temperature SG behavior including the disordered parent compound [38]. This suggests that the complex magnetic interactions between different spin states of $\mathrm{Co}^{3+}$ are responsible for the glassy behavior rather than evolution of $\mathrm{Co}^{4+}$ with Ti substitution [38]. However, Bos and Attfield studied the almost ordered AFM $\mathrm{Co}^{2+}$ $\mathrm{Nb}^{5+}$ double perovskite systems $(\mathrm{La} A) \mathrm{CoNbO}_{6}(A=\mathrm{Ca}, \mathrm{Sr}$, and $\mathrm{Ba}$ ) using magnetization and neutron powder diffraction measurements and report an enhancement in the geometrical frustration with increase in the ionic radii of $A$-site cations [7]. More recently, we studied the evolution of the antiferromagnetic insulating ground state in the $\mathrm{Sr}_{2-x} \mathrm{La}_{x} \mathrm{CoNbO}_{6}$ $(x=0-1)$ samples as a result of the enhancement in $\mathrm{Co}^{2+}$ concentration and hence $B$-site ordering with the La substitution [39] as well as with strain in thin films [40]. However, the evolution of the complex spin states of $\mathrm{Co}^{3+}$, ground magnetic state for the $x \leqslant 0.4$, and order of magnetic phase transition in the $x \geqslant 0.6$ samples remain unresolved [39].

Therefore, to systematically understand the effect of $B$ site ordering on the geometrical frustration and hence the magnetic ground state, we present the detailed analysis of specific-heat data of the $\mathrm{Sr}_{2-x} \mathrm{La}_{x} \mathrm{CoNbO}_{6}(x=0-1)$ samples, which have not been explored before. We observe a Schottky anomaly peak in the $x \leqslant 0.4$ samples due to the fine splitting (spin-orbit coupling and octahedral distortion) of the CF states of $\mathrm{Co}^{3+}$ ions, which gradually shift toward high temperature with $x$ as well as magnetic field. This indicates an increase in the splitting of the responsible energy states in either case. For the $x \geqslant 0.6$ samples, the specific-heat curves show clear $\lambda$-type peaks due to evolution of the AFM ordering as a consequence of the increased concentration of $\mathrm{Co}^{2+}$ ions. Further, the shift of the $\lambda$ peak to the lower temperature and a reduction in the peak jump with the magnetic field clearly indicate a second-order phase transition for the $x \geqslant 0.6$ samples. This is supported by the absence of the thermal hysteresis in the $C_{P}(T)$ curves recorded in heating and cooling modes. Moreover, the analysis of the Schottky anomaly in the case of the $x \leqslant 0.4$ samples and calculated values of magnetic entropy and $\lambda$-like peak jump in the case of the $x \geqslant 0.6$ samples indicate the presence of the discrete energy levels in these samples due to spin-orbit coupling and octahedral distortion. Also, the obtained values of the Lande $g$ factor indicate the presence of the HS state $\mathrm{Co}^{3+}$ close to the nonmagnetic LS state in the $x \leqslant 0.4$ samples. Further, the detailed analysis of field-cooled thermoremanent magnetization (TRM) and ac susceptibility data demonstrate the low-temperature cluster-glass behavior in the $x \leqslant 0.4$ samples, where the intercluster and spin-spin interaction strength decrease with $x$.

\section{EXPERIMENTAL}

Polycrystalline samples of $\mathrm{Sr}_{2-x} \mathrm{La}_{x} \mathrm{CoNbO}_{6}(x=0-1)$ were synthesized in a single phase by a solid-state route using stoichiometric amounts of $\mathrm{SrCO}_{3}, \mathrm{Co}_{3} \mathrm{O}_{4}, \mathrm{Nb}_{2} \mathrm{O}_{5}, \mathrm{La}_{2} \mathrm{O}_{3}$, and final sintering at $1300{ }^{\circ} \mathrm{C}$; more details of preparation and characterization can be found in Ref. [39]. The temperatureand magnetic-field-dependent heat capacity using relaxation technique (1.8-300 $\mathrm{K}$ and up to $9 \mathrm{~T}$ ), dc-magnetic susceptibility in both zero-field-cooled (ZFC) and field-cooled warming (FCW) modes, ac-susceptibility measurements at different excitation frequencies, and time-dependent magnetization $[M(\mathrm{t})]$ in different protocols (i.e., TRM and aging effect) have been performed using DynaCool Physical Property Measurement System from Quantum Design at KIT, Germany. For more details of the protocol of different measurements, we refer to the next section with their respective discussion.

\section{RESULTS AND DISCUSSION}

In Fig. 1(a), we show the zero-field-temperature-dependent specific-heat $\left[C_{P}(0, T)\right]$ data of $\mathrm{Sr}_{2-x} \mathrm{La}_{x} \mathrm{CoNbO}_{6} \quad(x=$ $0-1)$ samples recorded in the logarithmic steps from 


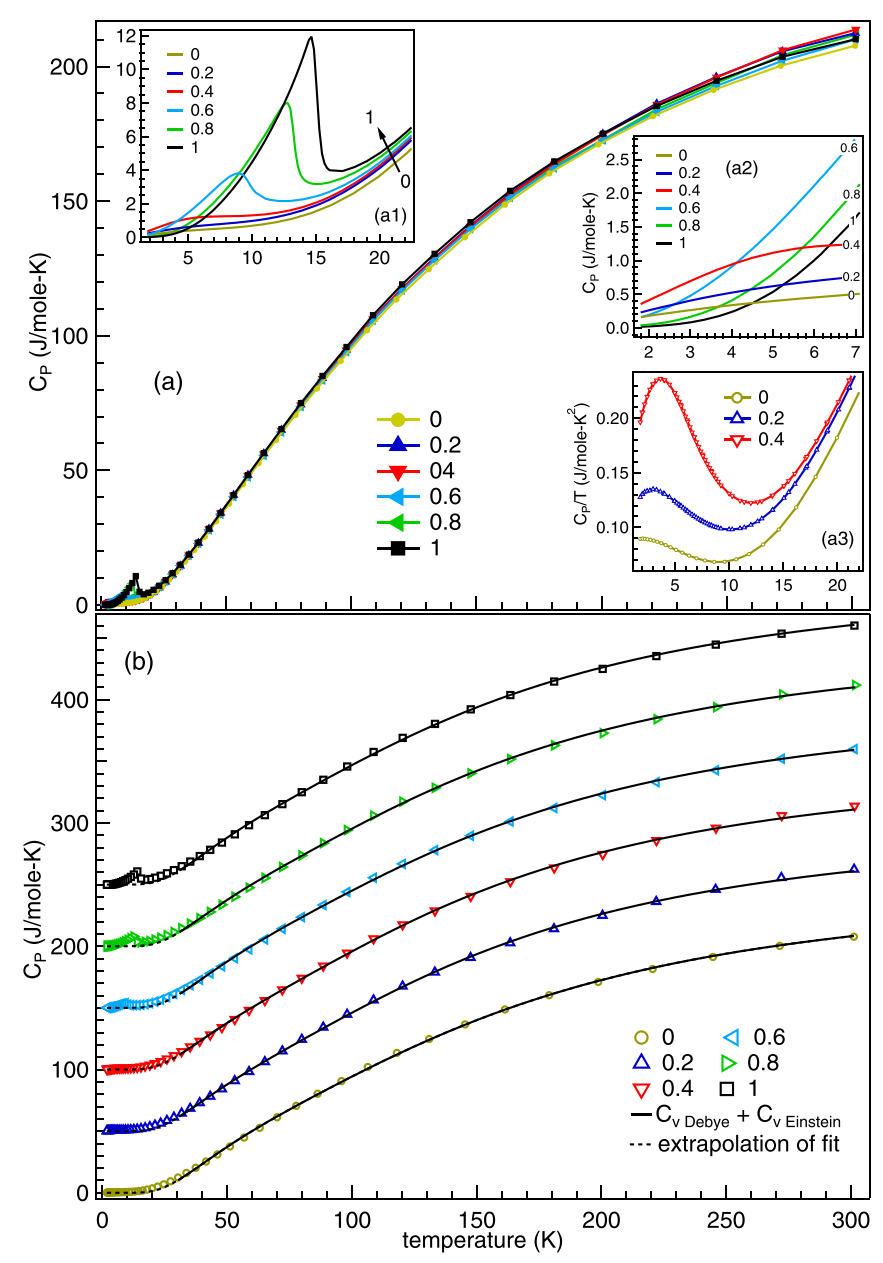

FIG. 1. (a) The temperature-dependent specific-heat curves of $\mathrm{Sr}_{2-x} \mathrm{La}_{x} \mathrm{CoNbO}_{6}(x=0-1)$ samples measured from $1.8 \mathrm{~K}$ to $300 \mathrm{~K}$ at zero magnetic field. Insets (a1) and (a2) show the enlarged view of the low-temperature region up to $23 \mathrm{~K}$ and $7 \mathrm{~K}$, respectively, (a3) shows the $C_{P} / T$ vs $T$ plot of low-temperature specific heat for the $x=0-0.4$ samples. (b) The best fit of the specific-heat data from 30-300 K using the combination of Einstein and Debye models (black solid lines). The dashed lines in the low-temperature region are the extrapolation of high-temperature fit. Each curve is vertically shifted cumulatively by $50 \mathrm{~J} / \mathrm{mole}-\mathrm{K}$ for the clear presentation.

1.8-300 K. The observed nonsaturating values of $C_{P}$ ( 208-214 J/mole-K) at $300 \mathrm{~K}$ for all samples are found to be less than the classical Dulong-Petit limit, i.e., $C_{V}=3 n R$ $=249.4 \mathrm{~J} / \mathrm{mole}-\mathrm{K}$, where $n$ and $R$ are the number of atoms per formula unit (ten in the present case) and molar gas constant, respectively [41]. We present the enlarged view of the low-temperature regime from $1.8 \mathrm{~K}$ to $\sim 23 \mathrm{~K}$ in the inset of Fig. 1(a1). A $\lambda$-like peak in the specific heat curves can be clearly observed for the $x \geqslant 0.6$ samples, which shifts to the higher temperature with a significant enhancement in the peak jump $\left(\Delta C_{P}\right)$ with the La substitution. We observe the peak maxima at $\sim 9.0(1), 12.8(1)$, and $14.7(1) \mathrm{K}$ and $\Delta C_{P}$ of $\sim 2.3,6.7$, and $10.0 \mathrm{~J} / \mathrm{mole}-\mathrm{K}$ from the lattice background (discussed later) for the $x=0.6,0.8$, and 1 samples, respectively. The evolution of this $\lambda$-like peak is attributed to the enhancement in the long-range AFM ordering below Néel temperature $\left(T_{\mathrm{N}}\right)$ for the $x \geqslant 0.6$ samples as also evident in the magnetization measurements presented in Ref. [39]. Note that $\mathrm{La}$ substitution at $\mathrm{Sr}$ site converts $\mathrm{Co}$ valence state from $3+(x=0)$ to $2+$ in the $x=1$ sample [39]. Interestingly, for the $x=1$ sample $\left[T_{\mathrm{N}}=14.7(1) \mathrm{K}\right]$, Co is present only in the $2+$ oxidation state and due to the weak $\mathrm{CF}, 28$-fold degenerate free-ion ${ }^{4} \mathrm{~F}$ term $(S=3 / 2 ; L=3)$ of $\mathrm{Co}^{2+}\left(3 d^{7}\right)$ splits into 12-fold degenerate ${ }^{4} T_{1 g}$ ground term with effective orbital angular momentum $(\tilde{L})=1$. This is further split by the spin-orbit coupling, resulting in the ground-state Kramers doublet with the pseudospin $(\tilde{S})=1 / 2[31,42]$. Here, the value of $\Delta C_{P}$ predicted for the mean-field AFM spin-wave ordering for $\mathrm{Co}^{2+}$ ( $\tilde{S}=1 / 2$ ) can be calculated using the equation below [43]:

$$
\Delta C_{P\left(T_{N}\right)}=5 R \frac{S(S+1)}{S^{2}+(S+1)^{2}} \approx 12.47 \mathrm{~J} / \text { mole }-\mathrm{K} .
$$

The close agreement between the experimental $\Delta C_{P}(10.0$ $\mathrm{J} /$ mole $-\mathrm{K}$ ) and the predicted value for the $\tilde{S}=1 / 2$ system in Eq. (1) indicates the persistence of the single $\mathrm{Co}^{2+}$-ion-like discrete energy levels with a ground-state doublet in these samples [31]. This is consistent with the observed twofold degenerate ground state of $\mathrm{Co}^{2+}$ ions in $\mathrm{Ba}_{2} \mathrm{CoUO}_{6}$ sample from the specific heat study [44]. On the other hand, a loss in the experimental $\Delta C_{P}$ suggests the presence of the short-range magnetic correlations above the $T_{N}$, which is a typical feature of magnetically frustrated systems $[7,9]$.

Furthermore, for the $x \leqslant 0.4$ samples, no such magnetic phase transition is observed down to $1.8 \mathrm{~K}$. However, lowtemperature specific heat increases with the La concentration from $x=0$ to 0.4 , as more clearly visible in the inset of Fig. 1(a2). It can be observed that specific heat at $2 \mathrm{~K}$ increases from $x=0$ to 0.4 and then decreases with further increase in the La substitution from $x=0.6$ to 1 . A broad Schottky anomaly due to the transition between low-lying electronic energy levels of $\mathrm{Co}^{3+}$ could be the possible reason for this [26,27], which is presented more clearly as $C_{P} / T$ versus $T$ plot in the inset of Fig. 1(a3). This indicates further splitting of the responsible high-/intermediate-spin (IS; $t_{2 g}^{5} e_{g}^{1}$ ) states of $\mathrm{Co}^{3+}$ due to the spin-orbit coupling and/or octahedral distortion in these samples [26-28]. Further, the position of the anomaly, which is the measure of the energy gap between the low-lying states, shifts to the higher temperature with the La substitution for $x \leqslant 0.4$ samples [see inset of Fig. 1(a3)]. This rules out the presence of weak ferromagnetism as the origin of this anomaly in these samples, because FM interactions suppress with the La substitution in $\mathrm{Sr}_{2-x} \mathrm{La}_{x} \mathrm{CoNbO}_{6}$ samples [39]. Also, an enhancement in the strength of this anomaly indicates the increase in the population of responsible IS/HS states with $x(\leqslant 0.4)$. However, with further increase in $x$ for the $x \geqslant 0.6$ samples, our analysis suggests the decrease in the strength of this Schottky peak due to the dominance of the $\mathrm{Co}^{2+}$ concentration and hence long-range AFM ordering. The possible origin of this Schottky anomaly and its evolution with the La substitution are discussed later.

Note that the measured specific heat in the present case can be mainly comprised of the lattice specific heat due to phonons $\left(C_{\text {latt }}\right)$, electronic specific heat due to conduction electrons $\left(C_{\mathrm{el}}\right)$, magnetic specific heat due to exchange interaction between the spins $\left(C_{\text {mag }}\right)$, and a Schottky-type anomaly due to the closely spaced electronic energy levels of Co cations $\left(C_{S}\right)$ as well as hyperfine splitting due to interaction 
TABLE I. The extracted parameters from the specific heat data of $\mathrm{Sr}_{2-x} \mathrm{La}_{x} \mathrm{CoNbO}_{6}$ by fitting from $30 \mathrm{~K}$ to $300 \mathrm{~K}$ using the combined Debye and Einstein models with $m=0.69$.

\begin{tabular}{lll}
\hline \hline$x$ & $\theta_{\mathrm{D}}(\mathrm{K})$ & $\theta_{\mathrm{E}}(\mathrm{K})$ \\
\hline 0 & $691(7)$ & $170(3)$ \\
0.2 & $687(6)$ & $168(3)$ \\
0.4 & $678(8)$ & $161(4)$ \\
0.6 & $693(8)$ & $168(3)$ \\
0.8 & $680(8)$ & $166(4)$ \\
1 & $677(8)$ & $167(3)$ \\
\hline \hline
\end{tabular}

between electronic and nonzero nuclear spins $(I=7 / 2)$ of ${ }^{59} \mathrm{Co}^{3+}\left(C_{\text {hyp }}\right)$, i.e., $C_{P}(T)=C_{\text {latt }}+C_{\mathrm{el}}+C_{\mathrm{mag}}+C_{\mathrm{S}}+$ $C_{\text {hyp. }}$. We first analyze the high temperature (from $30 \mathrm{~K}$ to $300 \mathrm{~K})$ specific-heat data, as shown in Fig. 1(b), considering only lattice contributions with the combination of Debye and Einstein heat-capacity models, where no other significant contributions are expected in these samples. The $C_{\text {latt }}$ can be expressed as $C_{\text {latt }}=m C_{\mathrm{V} \text { (Debye) }}+(1-m) C_{\mathrm{V} \text { (Einstein) }}$, where $m$ is the fractional contribution of the Debye model in the total lattice heat capacity, $C_{\mathrm{V} \text { (Debye) }}$ is the Debye lattice specific heat at constant volume, given as [45]

$$
C_{\mathrm{V}(\text { Debye })}(T)=9 n R\left(\frac{T}{\theta_{D}}\right)^{3} \int_{0}^{\theta_{D} / T} \frac{x^{4} e^{x}}{\left(e^{x}-1\right)^{2}} d x,
$$

and $C_{\mathrm{V}(\text { Einstein) }}$ is the Einstein specific-heat capacity at constant volume, given as [45]

$$
C_{\mathrm{V} \text { (Einstein) }}(T)=3 n R\left(\frac{\theta_{E}}{T}\right)^{2} \frac{e^{\theta_{E} / T}}{\left(e^{\theta_{E} / T}-1\right)^{2}} .
$$

Here we use single Debye $\left(\theta_{D}\right)$ and Einstein $\left(\theta_{E}\right)$ temperatures due to acoustic and optical phonons, respectively. The black solid lines in Fig. 1(b) represent the best fit from 30 to $300 \mathrm{~K}$ using the above equations. For the fitting, we use the Padé approximation function as developed by Goetsch et al. for the Debye specific heat function [46]. A combination of $\sim 69 \%$ Debye and $\sim 31 \%$ Einstein model results in the best fitting of the data and the extracted values of $\theta_{D}$ and $\theta_{E}$ are listed in Table I for all the samples. Here, the obtained values of the Debye temperature (670-700 K) are significantly higher as compared to the perovskite oxides $\mathrm{La}_{1-x} \mathrm{Sr}_{x} \mathrm{CoO}_{3}(400-500 \mathrm{~K})$ [47]. This is consistent with the nonsaturated specific-heat curves at $300 \mathrm{~K}$ (see Fig. 1) and indicates the higher associated thermal conductivity of these $\mathrm{Sr}_{2-x} \mathrm{La}_{x} \mathrm{CoNbO}_{6}$ samples, which suggest their possible use in the electronic devices [48]. Further, the electronic contribution in the specific heat can be written as $C_{\mathrm{el}}=\gamma T$, where $\gamma$ is the Sommerfeld coefficient and can be expressed in the free-electron model as $\gamma=\left(\pi^{2} k_{B}^{2} / 3\right) D\left(E_{F}\right)$, where $k_{B}$ and $D\left(E_{F}\right)$ are the Boltzman's constant and density of states at the Fermi energy for both spin directions, respectively [41]. We have tried the fitting by introducing the electronic contribution $(\gamma T)$ in the specific heat, but no improvement was observed. This is expected due to the insulating nature of these samples [39]. In Fig. 1(b), the dashed lines represent an extrapolation of the fitted curves, which show a significant deviation in the low-temperature range down to $1.8 \mathrm{~K}$, indicating the other dominating contributions like Schottky, hyperfine, magnetic, etc. in these samples.

Now we first focus on the analysis of the low-temperature Schottky anomaly present in $x \leqslant 0.4$ samples to probe lowlying energy levels, which can be helpful in understanding their complex magnetic and electronic properties [27,49,50]. The nuclear Schottky anomaly usually lies at very low temperatures $(<2 \mathrm{~K})$ due to the closely spaced hyperfine split energy levels. However, in the high-temperature regime where the Schottky peak decays, the $C_{\text {hyp }}$ can be approximated as $C_{\text {hyp }}=B_{-2} / T^{2}$, where $B_{-2}$ is the temperature-independent and field-dependent proportionality constant [51]. Further, at low temperatures the lattice specific heat can be expressed as $C_{\text {latt }}=B_{3} T^{3}$ [41], where the constant $B_{3}$ is related to the Debye temperature $\theta_{\mathrm{D}}$ as $B_{3}=12 \pi^{4} n R / 5 \theta_{\mathrm{D}}^{3}$. Note that in the absence of magnetic interactions, the plot of $C_{P}(T) / T$ vs $T^{2}$ should follow a conventional straight fit, considering only lattice and electronic contributions at low temperatures. However, due to the presence of Schottky anomaly in the $x \leqslant 0.4$ samples and a significant magnetic contribution (long-range AFM ordering) in the $x \geqslant 0.6$ samples, the $C_{P} / T$ vs $T^{2}$ curves are not straight in the present case (not shown) and therefore higher order harmonics are required in $C_{\text {latt }}$ to fit the data in $\leqslant 30 \mathrm{~K}$ temperature range. Here it is important to mention that the $C_{\text {latt }}$ estimated from the combined Debye and Einstein models deviates at the low temperatures and even a small error in the Padé approximation function can give the misleading information due to weak Schottky contribution in the $C_{P}(T)$ curves, particularly for the $x \leqslant 0.4$ samples. Therefore, considering the Schottky anomaly, we estimate lattice specific heat $(1.8-30 \mathrm{~K})$ for $x \leqslant 0.4$ samples using the harmonic lattice model up to the higher orders, i.e., $C_{\text {latt }}=B_{w} T^{w}, w=3,5,7$, 9 [52]. Further, the Schottky contribution in the specific heat can be expressed as [45]

$$
\begin{aligned}
C_{S}= & \frac{\rho R}{T^{2}}\left[\left(\frac{\sum_{i} g_{i} E_{i}^{2} \exp \left(-E_{i} / T\right)}{\sum_{i} g_{i} \exp \left(-E_{i} / T\right)}\right)\right. \\
& \left.-\left(\frac{\sum_{i} g_{i} E_{i} \exp \left(-E_{i} / T\right)}{\sum_{i} g_{i} \exp \left(-E_{i} / T\right)}\right)^{2}\right],
\end{aligned}
$$

where $\rho$ is the concentration of the contributing magnetic sites, and $E_{i}$ and $\mathrm{g}_{i}$ are the energy and degeneracy of the $i$ th level. Figure 2(a) shows the specific heat of the $x=0-0.4$ samples plotted as $C_{P} / T$ vs $T$ on the semilogarithmic scale to clearly represent the low-temperature $(1.8-20 \mathrm{~K})$ behavior. A two-level Schottky contribution used for the $\mathrm{Co}^{3+}$ ions in case of $\mathrm{LaCoO}_{3}$ [27] cannot fit the low-temperature broad peak observed in $x \leqslant 0.4$ samples and a three-level scheme (i.e., $E_{0}, E_{1}$, and $E_{2}$ ) has been employed for the best fit of the data in Fig. 2(a). Orlov et al. also observed an additional energy gap of $21.8 \mathrm{~K}$ in the Schottky anomaly of $\mathrm{Gd}_{0.4} \mathrm{Sr}_{0.6} \mathrm{CoO}_{3-\delta}$, which was associated with the presence of holes in the oxygen $2 p$ orbital [53]. However, our magnetization measurements indicate the complete filling of the oxygen $2 p$ orbital in the present case [39]. At the same time, high resolution near edge XAS measurements may be useful to probe the possibility of any marginal oxygen deficiency [54]. Further, an excess $C_{P}$ is observed at the very low temperature $(<3 \mathrm{~K})$, which indicates the presence of significant hyperfine contribution. 

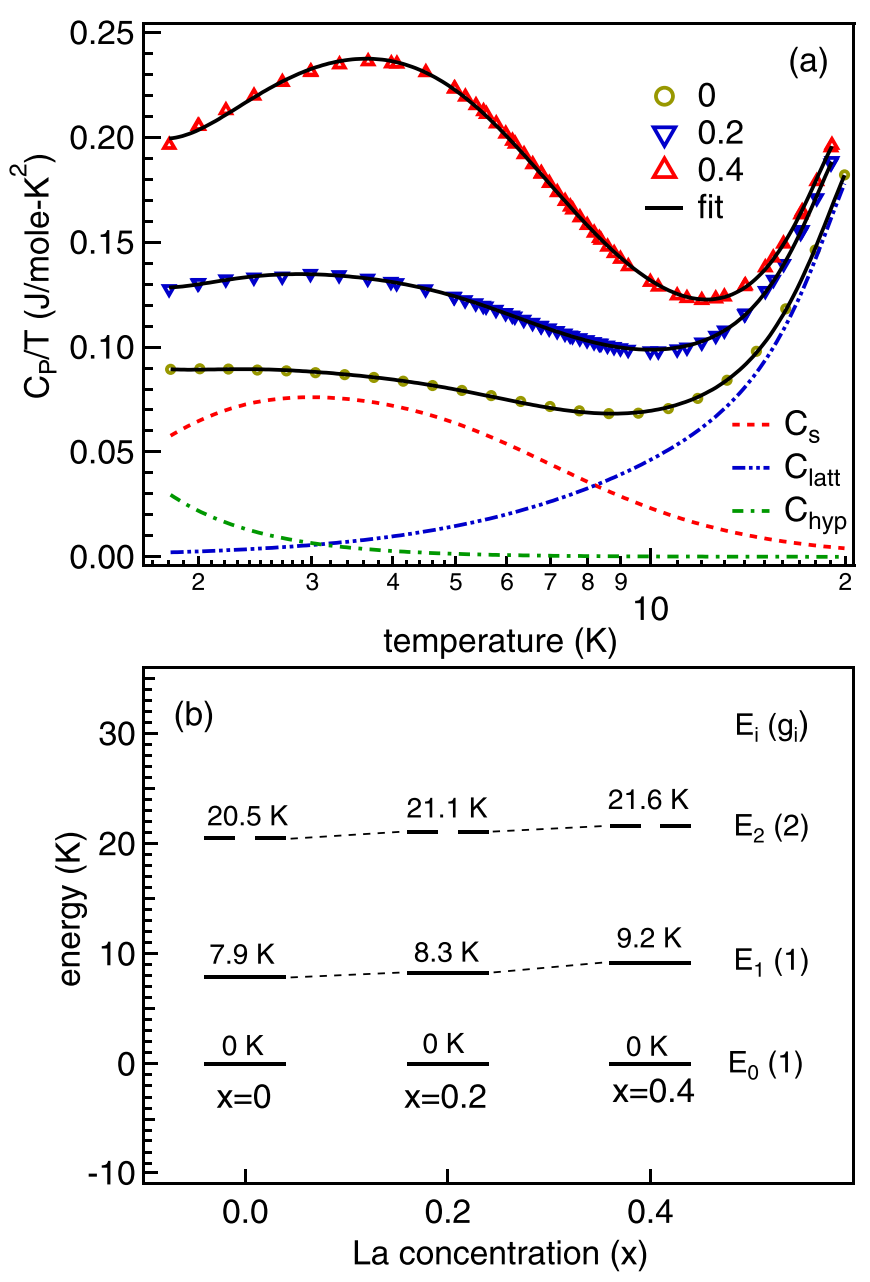

FIG. 2. (a) The low-temperature specific-heat data of the $x=0$ 0.4 samples from $1.8-20 \mathrm{~K}$ on the semilogarithmic scale as $C_{P} / T$ vs $T$ plot. The solid black lines represent the best fit of the data using the lattice, Schottky, and approximated hyperfine contributions. The dashed lines show the individual contributions in the $C_{P}(T)$ for the $x=0$ sample. (b) The proposed energy-level scheme as a function of $x$.

A similar observation is reported for around $T<1 \mathrm{~K}$ in the $\mathrm{La}_{1-x} \mathrm{Sr}_{x} \mathrm{CoO}_{3}$ samples due to the nonzero nuclear spins $(I=7 / 2)$ of ${ }^{59} \mathrm{Co}$ ions [47]. To estimate the $C_{\text {latt }}$, we first fit the $C_{P}(T)$ curves between $20-30 \mathrm{~K}$ using the harmonic lattice model for the initial choice of the constant parameters and finally allow all the free parameters to vary simultaneously. The solid lines in Fig. 2(a) represent the best fit of the data with the $C_{\text {latt }}, C_{\mathrm{S}}$, and $C_{\text {hyp }}$ contributions, where the dashed curves show the individual contributions in the $C_{P}(T)$ for the $x=0$ sample.

To understand the above fitting results, we note here that the triply orbital degenerate $t_{2 g}$ level of the $\mathrm{HS} \mathrm{Co}^{3+}$ with effective orbital moment $\tilde{L}=1$ can be further split by the spin-orbit coupling resulting in a low lying triplet $(\widetilde{J}=1)$, and first and second excited quintet $(\tilde{J}=2)$ and septet $(\tilde{J}=3)$, respectively [28]. Further, the octahedral distortion splits the spin-orbit triplet into a singlet and doublet, and their relative position depends on the nature of the distortion. For example, a tetragonal elongation $\left(\mathrm{O}_{4 h}\right)$ results in the low-lying singlet and an excited doublet, while tetragonal compression alters this energy scheme [29]. A large CF, on the other hand, can result into the reduction of the spin multiplicity of $\mathrm{Co}^{3+}$ and hence can push the LS state below/close to the HS state, see Refs. [25-29] for more details about the presence of the spin-orbit triplet of $\mathrm{HS} \mathrm{Co}^{3+}$ above the $\mathrm{LS}$ state in $\mathrm{LaCoO}_{3}$. In the present case, we obtain the best fit of the Schottky anomaly using three level contributions with a singlet ground and first excited states, and doublet second excited states, i.e., $g_{0}=$ $g_{1}=1, g_{2}=2$. Here, our analysis suggests that the spin-orbit triplet of the HS state (resulting in $E_{1}$ and $E_{2}=E_{3}$ in the low symmetry) is lying close to the LS state of $\mathrm{Co}^{3+}$, which is the origin of this additional energy state $\left(E_{0}\right)$ observed in these samples. This indicates a relatively weak CF in these samples as compared to the $\mathrm{LaCoO}_{3}$, where the LS state lies $\sim 10-15 \mathrm{eV}$ below the spin-orbit triplet of the HS state of $\mathrm{Co}^{3+}[25,26,28]$. This energy-level scheme very well reproduces the experimental $C_{P}(T)$ data, which clearly indicate the persistence of the discrete atomic energy states in these $x \leqslant$ 0.4 samples. The extracted energy levels from the best fit of the $C_{P}(T)$ curves are shown in Fig. 2(b) in the units of temperature, i.e., $E_{i} / k_{B}$, for $\rho=5.3(2) \%, 8.1(3) \%$, and $15.7(3) \%$ for the $x=0,0.2$, and 0.4 samples, respectively. This indicates that only a small fraction of $\mathrm{Co}^{3+}$ ions is in the HS state at low temperatures, which increases with $x$ as evident from the enhancement in the strength of the Schottky anomaly. Here, the observed shift in the Schottky anomaly toward higher temperatures with $x$ is possibly due to an enhancement in the octahedral distortion around the $\mathrm{Co}^{3+}$ ions. The substitution of smaller $\mathrm{La}^{3+}$ cations at the larger $\mathrm{Sr}^{2+}$ sites results in the enhancement of the $\mathrm{Co}^{2+}$ concentration, which is larger in size than $\mathrm{Co}^{3+}$ [37]. These two effects result in the lowering of the crystal symmetry $\left(\bar{d}_{A / A^{\prime}-O}=\sqrt{2} \bar{d}_{B / B^{\prime}-O}\right)$, causing the octahedral distortion, as also evident from the x-ray diffraction (XRD) and Raman spectroscopic measurements reported in Ref. [39].

For the detailed analysis of the Schottky peaks at low temperatures, we measured the high-resolution specific-heat data from $2 \mathrm{~K}$ to $30 \mathrm{~K}$ at different applied magnetic fields up to $\mu_{0} H=9 \mathrm{~T}$, as the $C_{P} / T$ vs $T$ plots are presented $(2-20 \mathrm{~K})$ in Figs. 3(a)-3(c) for the $x=0-0.4$ samples, respectively. Interestingly, a shift of the Schottky anomaly to the higher temperature and a notable reduction in the peak height can be clearly seen with increase in the magnetic field for the $x \leqslant 0.4$ samples. This reveals the magnetic origin of this anomaly, but the shift toward higher temperature with the field discards the long-range AFM ordering as the possible origin of this anomaly, which is also consistent due to increase of the $\mathrm{Co}^{2+}$ concentration with $x$. In Figs. 3(a)-3(c), we show the fitting of the $C_{P}(H, T)$ data between $2-20 \mathrm{~K}$ with the same procedure as mentioned above, while keeping the lattice contribution and concentration of the magnetic sites $(\rho)$ same as extracted from the $C_{P}(0, T)$ curves. The magnetic field lifts the degeneracy of the excited doublet (i.e., $E_{2} \neq E_{3}$ ) and the extracted best fit parameters are listed in Table II. In the inset of each panel of Fig. 3, we show the field-dependent Zeeman splitting of the zero-field doublet, which can be expressed as $\Delta(H)=\mathrm{g} \mu_{B} \mu_{0} H$, where $\Delta, \mathrm{g}$, and $\mu_{B}$ are the energy separation between the levels, Landé $g$ factor, and Bohr magneton, respectively. A linear fit of the field evolution of the Zeeman 


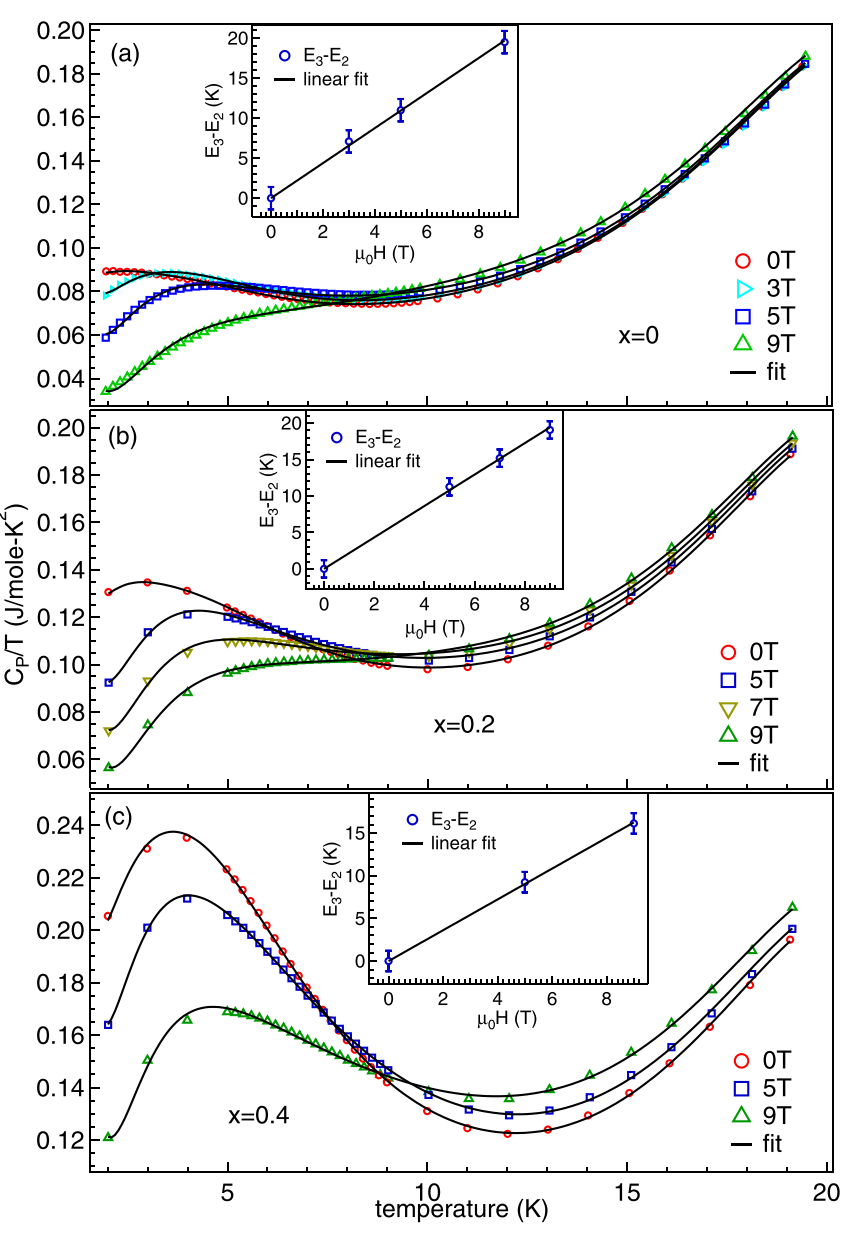

FIG. 3. (a)-(c) The temperature-dependent specific-heat data presented as $C_{P} / T$ vs $T$ plot measured at different magnetic fields for the $x=0-0.4$ samples, respectively. The solid black lines represent the best fit with the lattice, Schottky, and hyperfine contributions. Inset in each panel shows the field-dependent Zeeman splitting of the zero-field excited doublet.

splitting of the zero-field doublet (see insets in Fig. 3) gives $g=3.3(1), 3.2(2)$, and 2.7(1) for the $x=0,0.2$, and 0.4 samples, respectively. Here, it is important to note that $g$ value lies between $\sim 3-3.5$ for the HS state of $\mathrm{Co}^{3+}$, while $\mathrm{g} \sim 2$

TABLE II. The fitting parameters extracted from the field dependent Schottky anomaly for $x \leqslant 0.4$ samples $\left(E_{0}=0 \mathrm{~K}\right)$.

\begin{tabular}{lcrccc}
\hline \hline$x$ & $\mu_{0} \mathrm{H}(\mathrm{T})$ & $E_{1}(\mathrm{~K})$ & $E_{2}(\mathrm{~K})$ & $E_{3}(\mathrm{~K})$ & $\Delta=E_{3}-E_{2}$ \\
\hline 0 & 0 & 7.9 & 20.5 & 20.5 & 0 \\
& 3 & 8.9 & 18.1 & 25.2 & 7.1 \\
& 5 & 10.4 & 19.1 & 30.1 & 11 \\
& 9 & 13.1 & 26.4 & 45.9 & 19.5 \\
0.2 & 0 & 8.3 & 21.1 & 21.1 & 0 \\
& 5 & 10.5 & 19.4 & 30.7 & 11.3 \\
& 7 & 11.7 & 21.8 & 37.1 & 15.3 \\
& 9 & 13.0 & 26.0 & 45.1 & 19.1 \\
0.4 & 0 & 9.2 & 21.6 & 21.6 & 0 \\
& 5 & 10.5 & 20.7 & 30.0 & 9.3 \\
& 9 & 12.2 & 25.7 & 41.8 & 16.1 \\
\hline \hline
\end{tabular}

for the IS state, which further confirms the presence of the HS state of $\mathrm{Co}^{3+}$ close to the LS state at low temperatures. A small reduction in the $g$ value in the case of the $x=0.4$ sample is possibly due to the complex magnetic interactions resulting from the increase of $\mathrm{Co}^{2+}$ ions with La substitution and hence a direct probe of the low-lying energy levels using the inelastic neutron scattering would be useful to get further insight into [28]. Notably, we also observe a field-dependent minimal change in the low-lying nonmagnetic singlet states, which indicate the possibility of the partial breakdown of the pure ionic model. This can be due to the strong $\mathrm{CF}$ and/or covalent character in the bonding [40], which is also evident from the cluster-glass-like ground state for the $x \leqslant 0.4$ samples, discussed later.

Further, we extract the magnetic contribution in the specific heat by subtracting the lattice contributions from the $C_{P}(T)$ data using the harmonic lattice model. Figures 4(a)-4(c) show the low-temperature specific heat data of the $x=0-0.4$ samples along with the fitted curves between 20-30 K (indicated by vertical green arrows), which are extrapolated down to $1.8 \mathrm{~K}$. To find the magnetic part, the extrapolated curves were subtracted from the $C_{P}(T)$ data and the resulting magnetic specific heat curves are presented as $C_{\mathrm{mag}} / T$ vs $T$ on the left scale of Figs. 4(d)-4(f). We calculate the magnetic entropy as $S_{\text {mag }}(T)=\int_{T_{1}}^{T_{2}} \frac{C_{\mathrm{mag}}(T)}{T} \mathrm{~d} T$, where $T_{1}$ and $T_{2}$ are the lower and upper temperatures of interest. The $S_{\text {mag }}$ curves are shown on the right scale of Figs. 4(d)-4(f), which show the saturated values of $S_{\mathrm{mag}} \approx 0.6,0.9$ and $1.6 \mathrm{~J} / \mathrm{mole}-\mathrm{K}$ for the $x=0,0.2$ and 0.4 samples, respectively. We estimate the theoretical maximum entropy $S_{\text {theory }}=R \ln \Omega$, where $\Omega$ denotes the available quantum states, considering the composition weighted $\mathrm{Co}^{3+}$ with four energy states (up to $\sim 22 \mathrm{~K}$ ) and $\mathrm{Co}^{2+}$ ions with a doublet ground state, which are $11.53,10.37$, and $9.22 \mathrm{~J} / \mathrm{mole}-\mathrm{K}$ for the $x=0,0.2$, and 0.4 samples, respectively. Interestingly, the experimental $S_{\text {mag }}$ values are considerably lower than $S_{\text {theory }}$, which further suggest that only a small fraction of the $\mathrm{Co}^{3+}$ is in the higher magnetic state. For example, considering that only finite $\mathrm{HS}$ states of $\mathrm{Co}^{3+}$ ions are contributing in the experimental magnetic entropy, i.e., $S_{\text {mag }}=\rho R \ln \Omega$, we obtain $\rho \sim 5.2(2) \%$ for the $x=0$ sample. Importantly, the close agreement between this value of $\rho$ with that extracted from the Schottky anomaly further validate the four-level energy scheme employed in the present case. However, a small error in the determination of the lattice contribution due to the unavailability of the nonmagnetic reference analog cannot be completely neglected, as the zero-field-excited doublet of $\mathrm{Co}^{3+}$ is present up to $20-22 \mathrm{~K}$ for the $x \leqslant 0.4$ samples.

It is vital to investigate the nature of low-temperature AFM ordering observed in the $x \geqslant 0.6$ samples, and therefore we present high resolution $C_{P}(T)$ data in Figs. 5(a)-5(c) recorded from 2 to $30 \mathrm{~K}$ at various magnetic fields. Inset of Fig. 5(a) shows the enlarged view of the transition region. Interestingly, the $\lambda$-like peak shifts to the lower temperature and a significant reduction in the peak jump is observed with increase in the magnetic field for the $x \geqslant 0.6$ samples. This is a typical signature of the second order AFM-paramagnetic (PM) phase transition in complex oxides [55]. To further understand this field-dependent shift, the $\lambda$-peak position is plotted as a function of applied magnetic field for the $x=0.8$ and 1 samples 

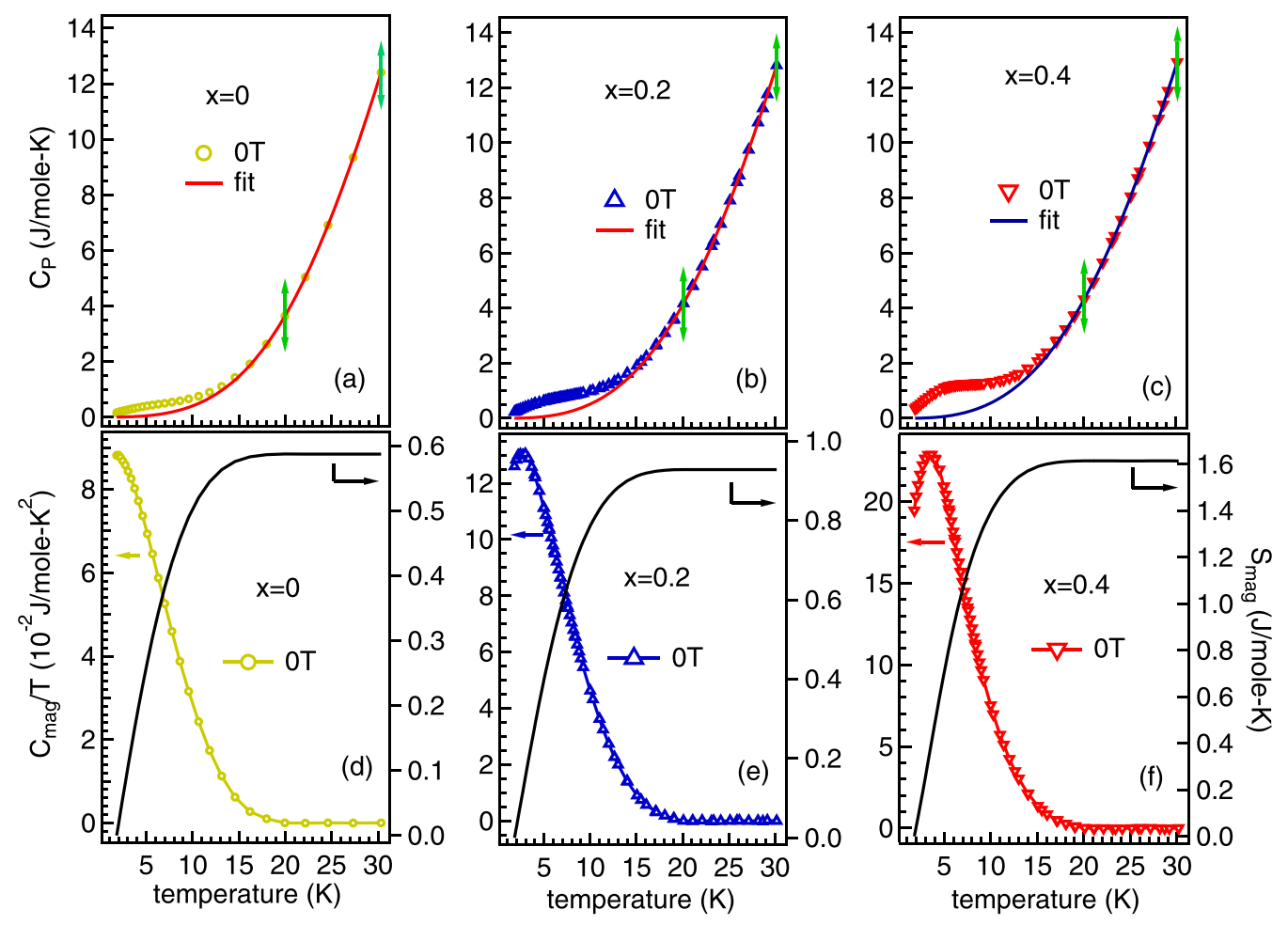

FIG. 4. (a)-(c) The low-temperature specific-heat data along with the lattice contribution (solid line) from the harmonic lattice model fitted between $20-30 \mathrm{~K}$ (marked by vertical green arrows). (d)-(f) The magnetic specific heat plotted as $C_{\mathrm{mag}} / T$ vs $T$ and magnetic entropy ( $\mathrm{S}_{\mathrm{mag}}$ ) on left and right scales, respectively, for the $x=0-0.4$ samples.

in the insets of Figs. 5(b) and 5(c), respectively. Here we estimate the decay of the AFM interactions with temperature and applied magnetic field as $H=H_{0}\left(1-T / T_{N}\right)^{\psi}$, where $H_{0}$ is the critical magnetic field required to break the AFM at $0 \mathrm{~K}$, see insets of Figs. 5(b) and 5(c). The obtained parameters from this analysis are $\psi=0.55(1), T_{N} \approx 12.8 \mathrm{~K}$, and $H_{0}=21.8(2)$ $T$ for the $x=0.8$ sample and $\psi=0.49(2), T_{N} \approx 14.7 \mathrm{~K}$, and $H_{0}=23.8(3) T$ for the $x=1$ sample. These values are consistent with those indirectly extracted from the magnetization measurements except for the critical magnetic-field values are found to be lower, for example, the $H_{0}=35(2) \mathrm{T}$ for the $x=$ 1 sample in Ref. [39].

To further confirm the order of magnetic phase transition in these samples, in Figs. 6(a)-6(c), we compare the $C_{P}(0, T)$ data recorded in both heating and cooling modes, which show no hysteresis in the vicinity of the magnetic transition that confirms the second-order AFM-PM phase transition in the $x \geqslant 0.6$ samples. We subtract the lattice contributions from the $C_{P}(T)$ data, as described above [see Figs. 6(d)-6(f)], and then plot the $C_{\mathrm{mag}} / T$ and $\mathrm{S}_{\mathrm{mag}}$ in Figs. 6(g)-6(i) in the lowtemperature range. It can be observed that $S_{\text {mag }}$ increases with the La concentration due to increase in the concentration of the ordered moments owing to an enhancement in the $\mathrm{Co}^{2+}$ concentration. A significantly high value of $C_{\mathrm{mag}} / T$ at $2 \mathrm{~K}$ in case of the $x=0.6$ sample, see Fig. $6(\mathrm{~g})$, possibly indicates the effect of Schottky anomaly present due to the $\mathrm{Co}^{3+}$ ions, which gradually suppresses with the $x$. Here, the saturation values of $S_{\mathrm{mag}}$ are found to be 2.8(1), 3.6(2), and 4.3(2) $\mathrm{J} /$ mole $-\mathrm{K}$ for the $x=0.6,0.8$, and 1 samples, respectively. To compare, we calculate the $S_{\text {theory }}=R \ln (2)$ due to the ground-state Kramers doublet $[42,44]$ of compositionweighted $\mathrm{Co}^{2+}$ ions only $(\tilde{S}=1 / 2)$, which are $S_{\text {theory }}=3.45$, 4.6, and $5.75 \mathrm{~J} / \mathrm{mole}-\mathrm{K}$ for the $x=0.6,0.8$, and 1 samples, respectively. The experimental $S_{\mathrm{mag}}$ values are found to be $\sim 81 \%, 78 \%$, and $74 \%$ of the $S_{\text {theory }}$ for these three samples, respectively. Here, a weak contribution of Schottky anomaly in $S_{\text {theory }}$ due to the presence of the $\mathrm{Co}^{3+}$ ions is not considered for the $x=0.6$ and 0.8 samples. Interestingly, the loss of around three-fourths of the total magnetic entropy during the phase transition indicates the presence of short-range magnetic correlations well above the transition temperature, which is expected for the magnetically frustrated compounds [7,9].

Notably, the spin-wave theory predicts that the lowtemperature magnetic specific-heat follows the relation $C_{\text {mag }} \propto T^{d / \phi}$, where $d$ is the dimensionality of the magnetic interactions and $\phi$ is the exponent in the dispersion relation $\omega \sim \mathrm{k}^{\phi}$, where $\phi=2$ for FM magnons and $\phi=$ 1 for AFM magnons and phonons [56]. The best fit of the low-temperature $C_{\text {mag }}$ data in the magnetically ordered state ( $T$ $<T_{N}$ ) for the $x=0.6-1$ samples gives $d=1.9(2), 2.3(1)$, and 2.7(2), respectively, for $\phi=1$ [see Figs. 7(a)-7(c)]. Here, this peculiar behavior indicates the transformation from $2 \mathrm{D}$ to $3 \mathrm{D}$ AFM spin wave ordering with increase in the La concentration from $x=0.6$ to 1 , which is possibly due to the inclusion of the Schottky anomaly at the low temperatures as a result of the HS Co ${ }^{3+}$ ions in the $x=0.6$ and 0.8 samples. This is also evident from the shape of the low-temperature $C_{P}$ curves of the $x \geqslant 0.6$ samples [see Fig. 1(a1)]. Therefore, we analyze the critical behavior of the $x=0.6-1$ samples near the AFM-PM phase transition by estimating the critical exponent 


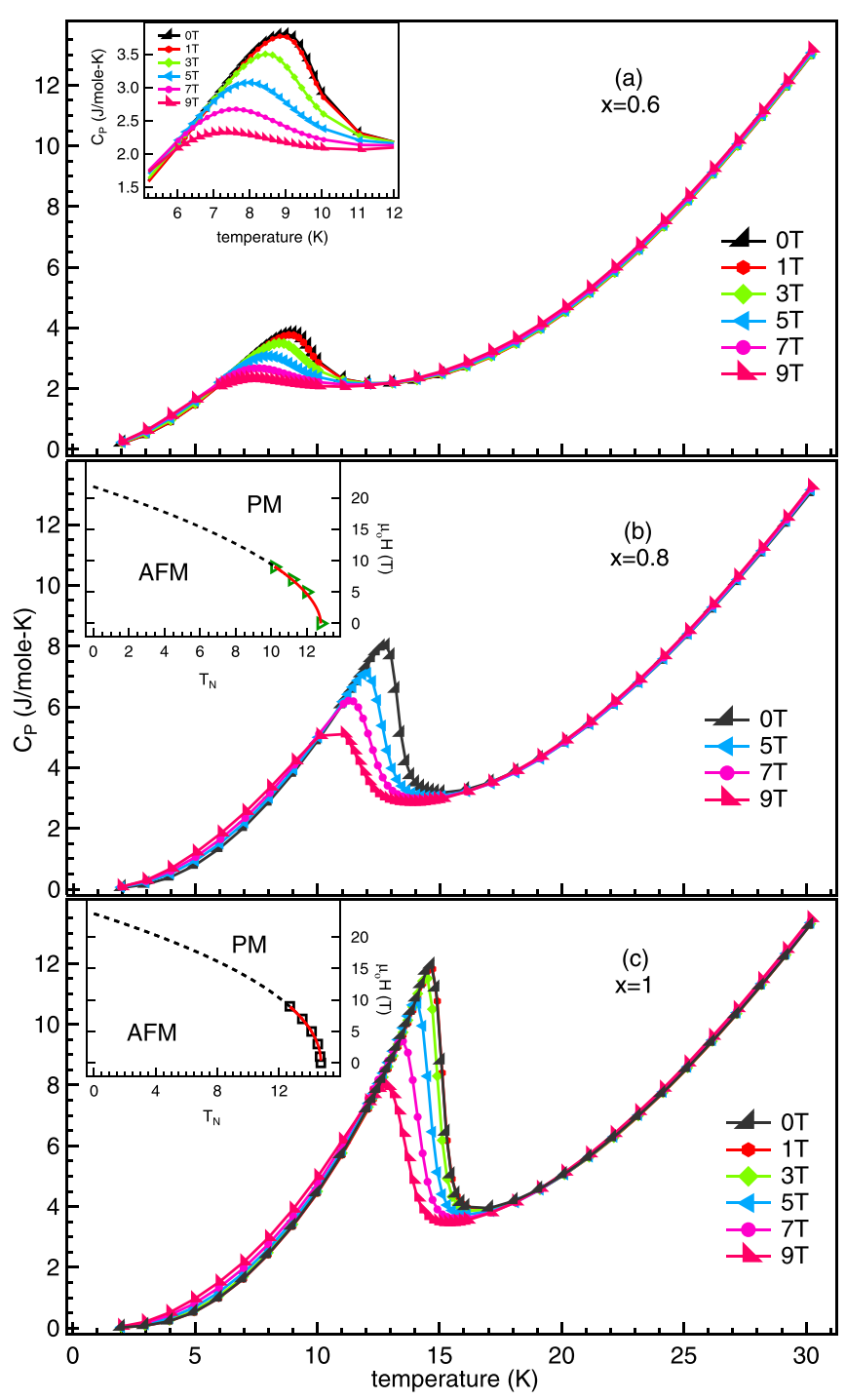

FIG. 5. (a)-(c) The temperature-dependent specific heat in the low-temperature region measured at different magnetic fields for the $x=0.6-1$ samples. Inset of (a) shows the enlarged view across the magnetic phase transition. Insets of (b) and (c) show the $H-T$ phase diagram extracted from the $C_{P}(H, T)$ data for the $x=0.8$ and 1 samples, respectively. The solid red lines represent the best fit using the AFM decay model and dashed black lines are the extrapolation of the fitted curves.

$\alpha$ from the $C_{P}(0, T)$ data, which can be expressed in the critical region as $C_{P}(T) \propto\left|T-T_{N}\right|^{-\alpha}$ and can be rewritten in the more realistic form as $[57,58]$

$$
C_{P}=B+C t+A^{ \pm}|t|^{\alpha}\left(1+E^{ \pm}|t|^{0.5}\right),
$$

where $t=\left(T-T_{N}\right) / T_{N}$ is the reduced temperature and $B, C$, and $E^{ \pm}$are the adjustable parameters. The superscripts + and - on $A$ and $E$ in Eq. (5) represent their values for $T$ $>T_{N}$ and $T<T_{\mathrm{N}}$, respectively. The linear term is the background contribution to the specific heat and last term is the anomalous contribution to the specific heat, where the factor within parentheses represents the scaling correction [57]. The best-fit parameters for the $x=0.6-1$ samples near the transition region are listed in Table III and resultant curves
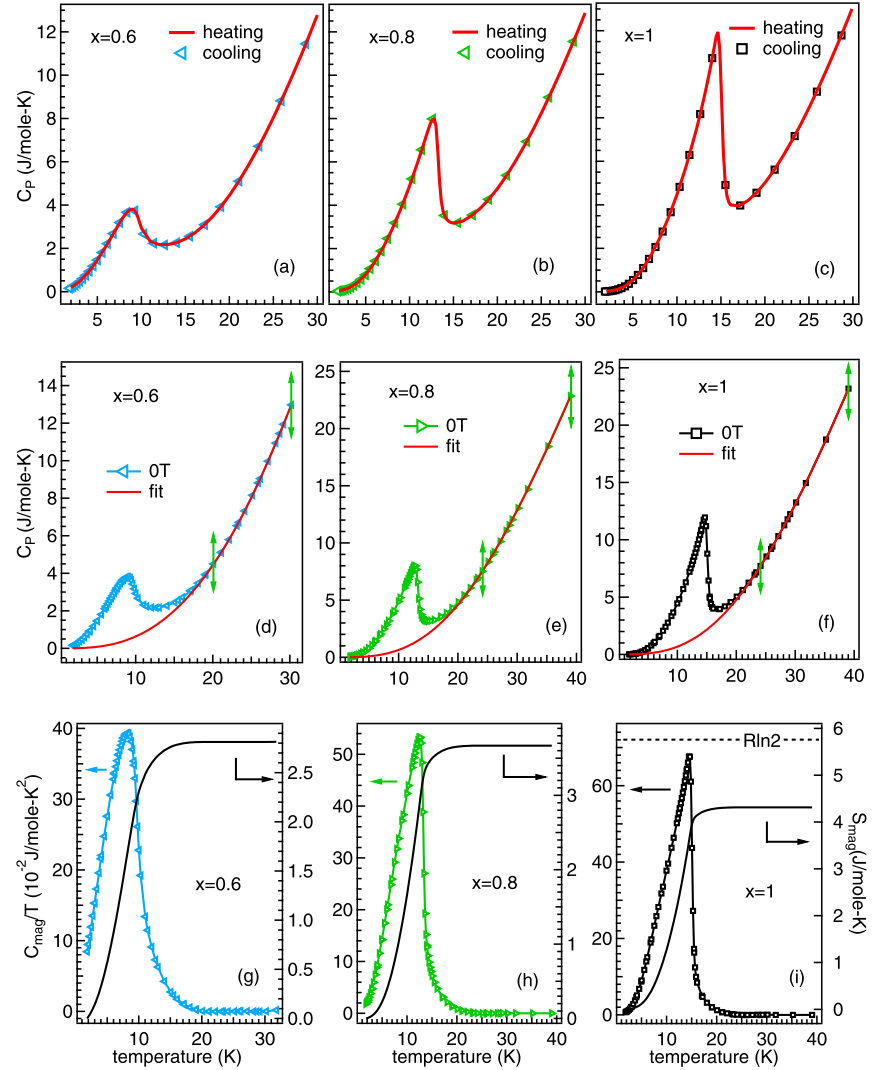

FIG. 6. (a)-(c) The zero-field specific-heat data measured in heating and cooling modes across the transition. (d)-(f) The specificheat data measured with smaller step size and fitted (solid red lines) using the harmonic lattice model in the region indicate by vertical green arrows and its extrapolation down to $1.8 \mathrm{~K}$. (g)-(i) The magnetic specific heat and magnetic entropy on the left and right scales, respectively for the $x=0.6-1$ samples. Dashed line in (i) represents the theoretical magnetic entropy expected for the groundstate Kramers doublet of $\mathrm{Co}^{2+}$ ions in the $x=1$ sample.

are shown by the continuous red lines in the Figs. 7(d)-7(f). To fit the data with Eq. (5) around the critical region, we first select the range very close to the transition temperature and avoid the rounding part, without introducing the scaling correction factor $(E)$. Then, we gradually increase the data points including the rounding part and get new set of the fitting parameters and allow the $E$ parameter to vary to improve the fit and finally the $T_{N}$ was varied to confirm the reliability of the fit. We use a similar procedure for fitting the data in both $T<T_{\mathrm{N}}$ and $T>T_{N}$ regions, separately. The values of critical exponents $\alpha$ and amplitude ratio, $\mathrm{A}^{+} / \mathrm{A}^{-}$, obtained for all the samples $(x \geqslant 0.6)$, see Table III, are close to the 3D Heisenberg model $\left(\alpha=-0.115\right.$ and $\left.\mathrm{A}^{+} / \mathrm{A}^{-}=1.58[59,60]\right)$ for the isotropic antiferromagnets. In this case, the effect of Schottky anomaly on the fitting parameters is not expected due to small temperature range in the critical region only. Therefore, a 3D type AFM ordering is anticipated for all $x \geqslant$ 0.6 samples.

As discussed above, the change in the ground-state energy with the applied magnetic field and presence of the bifurcation between in the ZFC-FCW curves of the magnetization data [39] indicates the possibility of low-temperature magnetic 

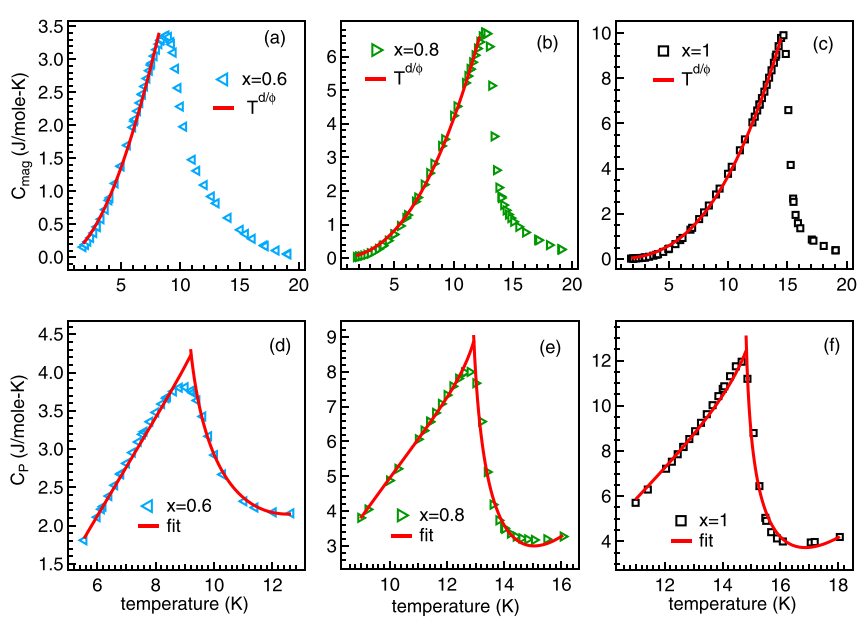

FIG. 7. (a)-(c) The magnetic specific-heat data of the $x=0.6-1$ samples along with the temperature dependence of the spin wave ordering (solid red lines) in the AFM region. (d)-(f) The zero-field specific-heat data of the $x=0.6-1$ samples in the vicinity of the transition temperature along with the best fit (solid red lines) in both AFM and PM regions using Eq. (5).

correlations in $x \leqslant 0.4$ samples. The magnetization and magnetocaloric studies show the low-temperature mixed FM and AFM interactions in the $x \leqslant 0.4$ samples [39]. This, along with the mixed valence and spin states of Co and resulting complex magnetic interactions between them, give rise to the magnetic frustration in these samples [39]. Therefore, it is important to investigate the complex spin dynamics and ground magnetic state in the $x \leqslant 0.4$ samples. To understand that, we first perform the dc magnetization measurements on the $x=$ 0.4 sample in both ZFC and FCW modes in which we cool down the sample to $2 \mathrm{~K}$ in the zero and at different applied magnetic fields, respectively, and then record the magnetization during the warming in both cases, as shown in Fig. 8. A magnetic irreversibility between ZFC and FCW curves is clearly observed below $T_{\text {irr }} \sim 7 \mathrm{~K}$ for $10^{2}$ Oe field, which shifts to the lower temperature with increase in the magnetic field as indicated by the arrow in Fig. 8. This field-dependent shift in the $T_{\text {irr }}$ is the typical signature of the presence of SG or superparamagnetic (SPM) state in these samples [33]. Thus, to further pinpoint this issue, we perform the field-cooled TRM

TABLE III. The critical exponent along with the other fitting parameters for the $x=0.6-1$ samples, extracted from the best fit of data above and below the transition temperature, as shown in Figs. 7(d)-7(f), using Eq. (5).

\begin{tabular}{lrrr}
\hline \hline & $x=0.6$ & $x=0.8$ & \multicolumn{1}{c}{$x=1$} \\
\hline$\alpha$ & $-0.11(2)$ & $-0.12(2)$ & $-0.11(1)$ \\
$\mathrm{A}^{+} / \mathrm{A}^{-}$ & $1.35(8)$ & $1.54(6)$ & $1.68(7)$ \\
$\mathrm{B}(\mathrm{J} /$ mole K $)$ & $8(1)$ & $33(5)$ & $40(5)$ \\
$\mathrm{C}(\mathrm{J} /$ mole K $)$ & $9(1)$ & $28(3)$ & $28(1)$ \\
$\mathrm{A}^{+}(\mathrm{J} /$ mole K $)$ & $-5.2(3)$ & $-47(1)$ & $-59(1)$ \\
$\mathrm{E}^{-}$ & $-0.78(6)$ & $-0.85(5)$ & $-0.55(3)$ \\
$\mathrm{E}^{+}$ & $1.8(2)$ & $0.01(1)$ & $-0.13(1)$ \\
$\mathrm{T}_{N}(\mathrm{~K})$ & $9.4(4)$ & $13.1(3)$ & $15.2(3)$ \\
\hline \hline
\end{tabular}

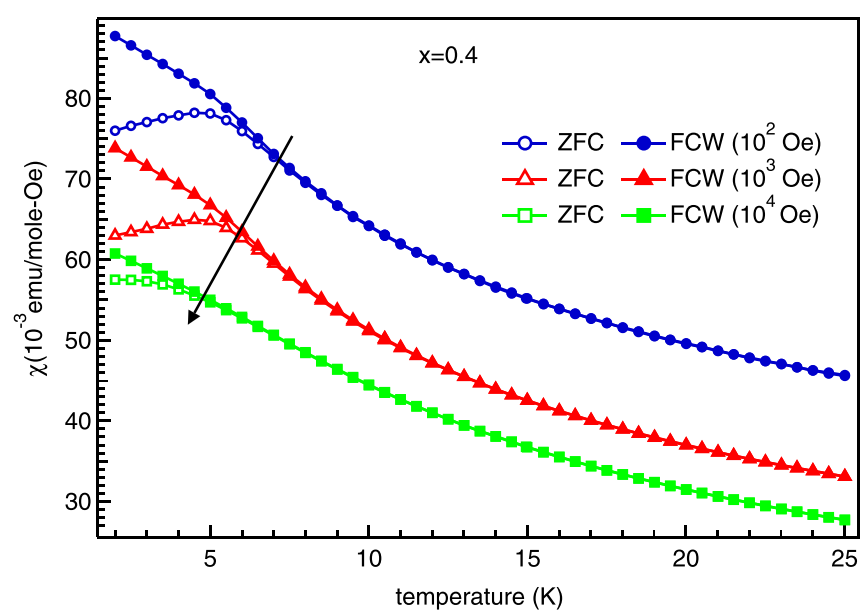

FIG. 8. The temperature-dependent dc susceptibility in ZFC and FCW modes measured at different applied magnetic fields for the $x=0.4$ sample. The curves recorded at $10^{2}$ and $10^{3}$ Oe are vertically shifted by $17 \times 10^{-3}$ and $5 \times 10^{-3} \mathrm{emu} /$ mole-Oe, respectively, for clarity in the presentation.

measurements on the $x=0-0.4$ samples at $2 \mathrm{~K}$ for different waiting times $\left(t_{w}\right)$, with the applied magnetic field of 1000 Oe, as shown in Figs. 9(a)-9(c). To record the TRM data, we first cool down the sample from 300 to $2 \mathrm{~K}$ in the presence of 1000 Oe magnetic field at a rate of $\sim 10 \mathrm{~K} / \mathrm{min}$ and then after a certain waiting time $t_{w}$ at $2 \mathrm{~K}$ the magnetic field was reduced to zero (with $220 \mathrm{Oe} / \mathrm{s}$, the highest available rate) and immediately start the magnetization measurement as a function of time $[M(\mathrm{t})]$. We record the data using the same protocol for the different waiting times, i.e., $\mathrm{t}_{w}=10^{2} \mathrm{~s}, 10^{3} \mathrm{~s}$, and $10^{4} \mathrm{~s}$, where sample was heated up to $50 \mathrm{~K}$ after each measurement in order to avoid any possibility of the remanence. The effect of waiting time and the slow decay of the remanent magnetization is clearly observed in the time evolution of the magnetization curves even for significantly high applied magnetic field value [see Figs. 9(a)-9(c)], which indicate the robust spin dynamics in these samples (see Fig. 1 and Table 1 of Ref. [61] for the TRM data on $x=0.4$ sample at different fields). The insets in each panel show the semilogarithmic plot of the data for $t_{w}=10^{3} \mathrm{~s}$ and solid red lines represent the best fit using the logarithmic relaxation model, $M(t)=M_{0}-r \log (t)$, where $M_{0}$ and $r$ are the spontaneous magnetization and relaxation rate, respectively [68]. From this fitting, the obtained parameters are $M_{0}=6.94(1), 10.84(2)$, and $12.13(1) \mathrm{emu} / \mathrm{mole}$ and $r=0.13(1), 0.30(1)$, and 0.74(2) for the $x=0,0.2$, and 0.4 samples, respectively. However, a significant deviation in the logarithmic decay model can be seen at the lower $t$ values, see insets in Fig. 9. Therefore, we fit the data using the well-known stretched exponential model given as [33]

$$
M(t)=M_{0}+M_{\mathrm{SG}} \times \exp \left[-\left(\frac{t}{t_{r}}\right)^{(1-\eta)}\right],
$$

where $M_{0}, M_{\mathrm{SG}}, \mathrm{t}_{r}, \eta$ are the FM and SG components of the magnetic moment, mean relaxation time, and relaxation rate, respectively. The best fit curves for the $x \leqslant 0.4$ samples are shown by the solid lines in Figs. 9(a)-9(c) and the extracted 


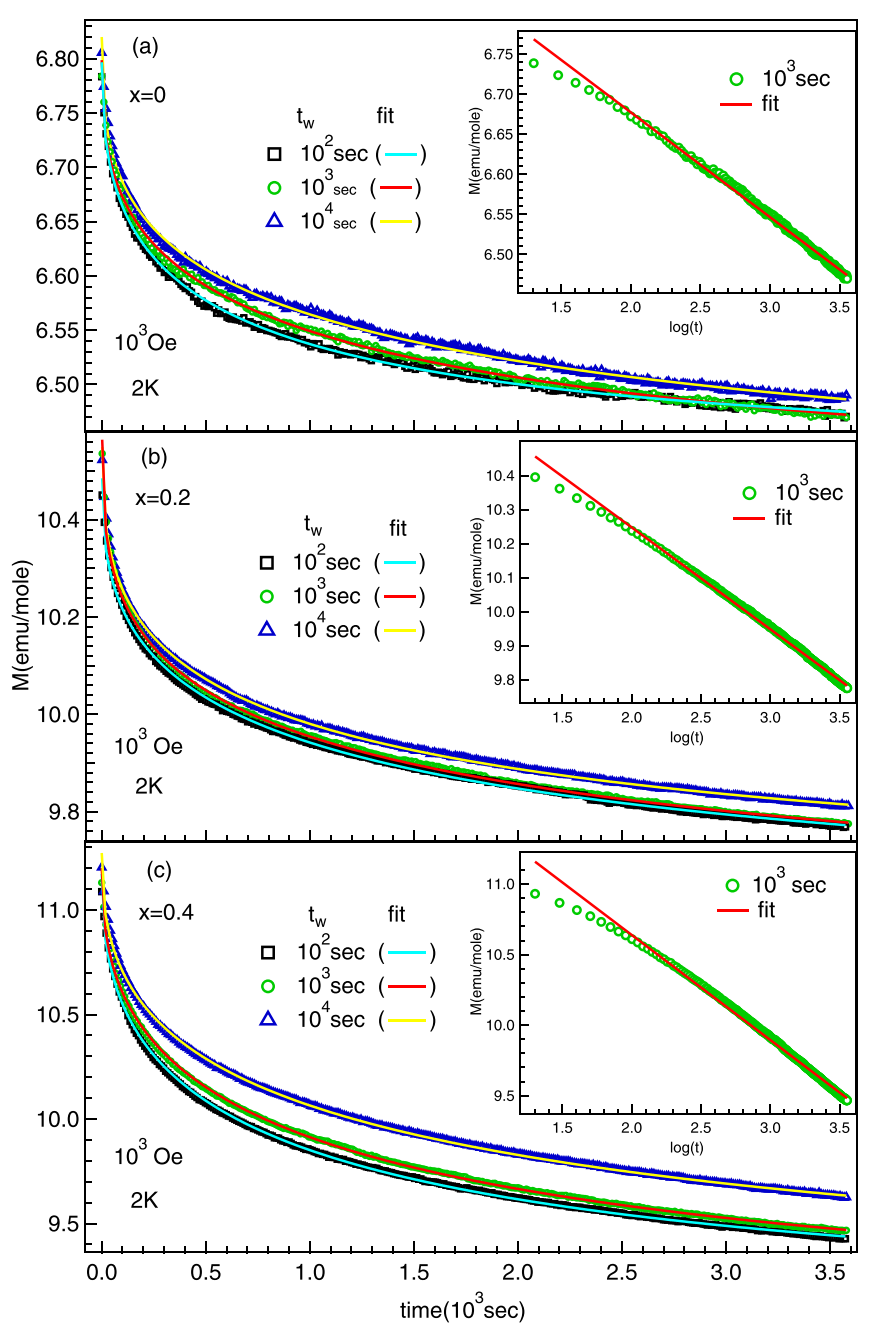

FIG. 9. (a)-(c) The field-cooled thermoremanent magnetization (TRM) data at $2 \mathrm{~K}$ for different waiting times $\left(\mathrm{t}_{w}=10^{2} \mathrm{~s}, 10^{3} \mathrm{~s}\right.$, and $10^{4} \mathrm{~s}$ ) with the applied magnetic field of $10^{3}$ Oe for the $x=0-0.4$ samples, respectively. The solid lines indicate the best fit of the data using the stretched exponential behavior. Insets in each panel show the semilogarithmic plots of the data for $\mathrm{t}_{w}=10^{3} \mathrm{~s}$ and solid red lines are the fit using the logarithmic relaxation model.

TABLE IV. The fitting parameters of the TRM data for the $x=$ 0-0.4 samples using Eq. (6) measured at 1000 Oe for different waiting times, $M_{0}$ and $M_{\mathrm{SG}}$ are in emu/mole.

\begin{tabular}{lcccrc}
\hline \hline & $\begin{array}{c}\mathrm{t}_{w} \\
(\mathrm{sec})\end{array}$ & $M_{0}$ & $M_{\mathrm{SG}}$ & $\begin{array}{c}\mathrm{t}_{r} \\
(\mathrm{sec})\end{array}$ & $\eta$ \\
\hline 0 & $10^{2}$ & $6.44(1)$ & $0.36(1)$ & $581(11)$ & $0.559(6)$ \\
& $10^{3}$ & $6.38(1)$ & $0.42(1)$ & $1280(60)$ & $0.597(6)$ \\
& $10^{4}$ & $6.37(1)$ & $0.45(1)$ & $1552(105)$ & $0.622(7)$ \\
0.2 & $10^{2}$ & $9.58(1)$ & $0.91(1)$ & $1248(48)$ & $0.590(6)$ \\
& $10^{3}$ & $9.53(1)$ & $1.03(1)$ & $1362(59)$ & $0.630(5)$ \\
& $10^{4}$ & $9.63(6)$ & $0.92(1)$ & $1116(32)$ & $0.603(4)$ \\
0.4 & $10^{2}$ & $9.09(1)$ & $2.08(2)$ & $987(21)$ & $0.549(5)$ \\
& $10^{3}$ & $9.02(1)$ & $2.18(2)$ & $1299(29)$ & $0.554(4)$ \\
& $10^{4}$ & $9.10(2)$ & $2.18(2)$ & $1635(52)$ & $0.571(4)$ \\
\hline \hline
\end{tabular}

parameters are listed in Table IV. The values of $t_{r}(>1000 \mathrm{~s})$ and $\eta(0<\eta<1)$ for all the three samples are typically in the range for the SG systems [69]. However, nonzero values of both $M_{0}$ and $M_{\mathrm{SG}}$ in Table IV indicate the coexistence of ferromagnetic and SG components in these samples. These parameters extracted from the TRM measurements along with those from aging effect (see Figs. 2 and 3 and Tables 2 and 3 in Ref. [61]), ruled out the possibility of the low temperature SPM state in the $x \leqslant 0.4$ samples.

In this context, to further investigate the low-temperature SG behavior, we performed ac-susceptibility measurements at 5 Oe ac magnetic field with different excitation frequencies $(f)$ below $30 \mathrm{~K}$, as shown in Fig. 10. A cusp in the real part $\left(\chi_{\mathrm{ac}}^{\prime}\right)$ and the corresponding peak in the imaginary part $\left(\chi_{\mathrm{ac}}^{\prime \prime}\right)$ of the ac susceptibility $\left(\chi_{\mathrm{ac}}=\chi_{\mathrm{ac}}^{\prime}+i \chi_{\mathrm{ac}}^{\prime \prime}\right)$ are clearly observed for the $x=0-0.4$ samples, which shifts to the lower temperature with increase in the La concentration [see Figs. 10(a)-10(f)]. For the $x=0-0.4$ samples, we observe that peak position shifts to the higher temperature and its amplitude decreases as we increase the frequency of the excitation ac field, which are the typical signatures of the SG ordering below the freezing temperature $\left(T_{f}\right)$ [70]. However, both these effects become less prominent with increase in the La concentration from $x=0$ to 0.4 . On the other hand, the $\chi_{\mathrm{ac}}^{\prime}$ data do not show any shift in the peak position with the excitation frequency for the $x=0.6$ and 1 samples, as shown in Figs. 10(g) and 10(h), respectively. Further, there is no long-time spin relaxation mechanism observed for $x \geqslant 0.6$ samples, see Fig. 4 for $x=1$ in Ref. [61], indicating the pure AFM state in these samples. For the $x \leqslant 0.4$ samples, we use in-phase component $\left(\chi_{\mathrm{ac}}^{\prime}\right)$ to study the effect of the applied frequency $(f)$ on the freezing temperature $\left(T_{f}\right)$, which is useful to understand their low temperature spin dynamics. In order to precisely assign the $T_{f}(f)$, we subtract a linear reference line from all the $\chi_{\mathrm{ac}}^{\prime}(T)$ curves, touching the highest frequency cusp at the two ends, as shown by the dotted lines in the insets of Figs. 10(a), 10(c), and 10(e) for the $x=0,0.2$, and 0.4 samples, respectively. In this process, the absolute value of the peak position of the $\chi_{a c}^{\prime}(T)$ curves may slightly vary due to change in the projection. However, relative shift in the peak position with the excitation frequency of the applied ac magnetic field is expected to remain invariant, which is used for the further analysis. After the linear reference line subtraction, the real components of the ac susceptibility data $\chi_{\mathrm{ac}}^{\prime}(T)$ are shown in Figs. 11(a)-11(c) for the $x=0-0.4$ samples, respectively. Note that the linear reference lines are part of the signal, which have been subtracted only to estimate the peak position by fitting the $\chi_{\mathrm{ac}}^{\prime}(T)$ curves using the Gaussian function near the freezing temperature, as indicated by the dotted curves in Figs. 11(a)-11(c). We use the extracted values of $T_{f}$ to calculate the Mydosh parameter, $\partial T_{f}=\Delta T_{f} / T_{f} \Delta\left(\log _{10} \omega\right)$, where $\Delta T_{f}$ is the frequency dependent shift in the freezing temperature and $\omega=2 \pi f$ is the angular frequency. The calculated values of $\partial T_{f}$ for the $x=0-0.4$ samples are given in Table $\mathrm{V}$ for the lowest and highest frequencies (i.e., 1001 and $8995 \mathrm{~Hz}$ ), where we choose $T_{f}$ as the freezing temperature at $1001 \mathrm{~Hz}$ excitation frequency. For the canonical SG systems the value of $\partial T_{f}$ lies between $0.005-0.01$, while for cluster glasses it lies between $\sim 0.03-0.08$, and $\partial T_{f}>0.2$ for the SPM systems owing to the high sensitivity of $T_{f}$ to the excitation frequency 

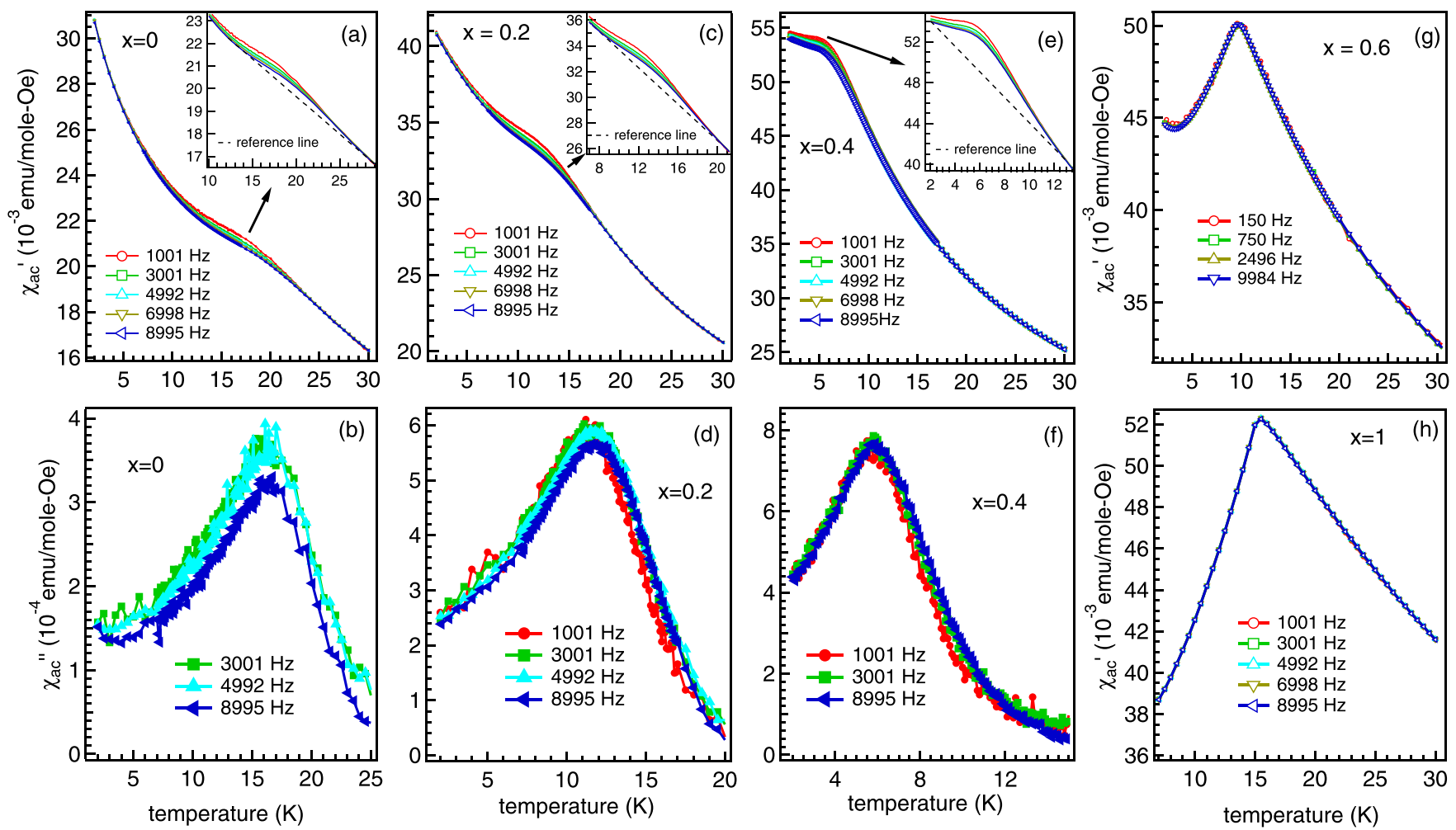

FIG. 10. The temperature-dependent real and imaginary parts of ac susceptibility measured at different frequencies with 5 Oe excitation magnetic field for the $x=0$ (a), (b), 0.2 (c), (d), and 0.4 (e), (f) samples. The real part of ac susceptibility for the $x=0.6$ and 1 samples are shown in (g) and (h), respectively. The insets in (a), (c), and (e) show zoomed view across the transition along with the dashed reference line subtracted for further analysis to be shown in Figs. 11(a)-11(c) for the $x=0-0.4$ samples, respectively.

[71,72]. Here, the calculated values of $\partial T_{f}$ are typically in the range for the insulating cluster-glass compounds [71].

The low temperature spin dynamics in these samples can be further investigated by analyzing the frequency dependence of $T_{f}$ following the critical slowing down model, i.e., using the equation below $[71,73]$ :

$$
\tau=\tau_{0}\left(\frac{T_{f}}{T_{\mathrm{SG}}}-1\right)^{-z v},
$$

where $\tau$ is the relaxation time corresponding to the excitation frequency $f$ (i.e. $\tau=1 / f$ ), $\tau_{0}$ is the characteristic relaxation time for a single spin flip, $T_{\mathrm{SG}}$ is the freezing temperature in the limit of zero excitation frequency where $\tau$ diverges, and $z v$ is the dynamical critical exponent, which is related to the spin-spin correlation length $(\xi)$ as $\xi=\left(T_{f} / T_{\mathrm{SG}}-1\right)^{-v}$ and $\tau \sim \xi^{z}$ [71,73]. In Figs. 11(d)-11(f), we show the best fit of the data using equation 8 for the $x=0-0.4$ samples, respectively and the extracted parameters are listed in Table V. The value of $z v$ lies between 4-12 for SG systems, while $z v=2$ for the conventional phase transitions [74,75]. The calculated values of $z v$ clearly demonstrate the SG-like ordering for all the three samples, which is expected to decrease with the La substitution as the system is leading towards the conventional AFM phase transition. However, a slight inconsistency in the $z v$ value is possibly due to the deviation from the ideal critical slowing down model for the $x=0.2$ sample. Further, for the ideal canonical spin glass systems with non-interacting spins the value of $\tau_{0}$ lies between $10^{-12}-10^{-14} \mathrm{~s}$, however, for the cluster-glass systems $\tau_{0}$ falls between $10^{-7}-10^{-10} \mathrm{~s}[76,77]$.
Here, the large values of the characteristic relaxation time, $\tau_{0}$ for all the samples as given in Table $\mathrm{V}$ indicate the slow spin dynamics due to the strong spin-spin correlations, which confirm the cluster-glass-like ground state in these samples. On the other hand, a decrease in the $\tau_{0}$ value with the La substitution indicates the reduction in the spin-spin correlations strength. Therefore, we fit the $T_{f}(\omega)$ data using the Vogel-Fulcher law, as given below [71,74]:

$$
\omega=\omega_{0} \exp \left(\frac{E_{a}}{k_{\mathrm{B}}\left(T_{f}-T_{0}\right)}\right),
$$

where $k_{B}$ is the Boltzmann's constant, $\omega_{0}$ is the characteristic frequency $\left(\omega_{0}=2 \pi / \tau_{0}\right), T_{0}$ is the Vogel-Fulcher temperature, which is the measure of the strength of the inter-cluster interactions, and $E_{a}$ is the average thermal activation energy. Here, we fix $\omega_{0}=2 \pi / \tau_{0}$ for all the samples and fit the data keeping $T_{0}$ and $E_{a}$ as the free parameters. The slope and intercept of $T_{f}$ vs $1 / \ln \left(\omega_{0} / \omega\right)$ plots give $E_{a} / k_{B}$ and $T_{0}$, respectively, as shown in the Figs. 11(g)-11(i) for the $x=0-0.4$ samples and the best fit parameters are listed in Table V. Interestingly, we found nonzero values of the $T_{0}$ for all samples, which indicate the interactions between the spins, and confirm the presence of cluster-glass-like ground state in these samples. A decrease in the value of $T_{0}$ indicates the tendency of reduction in the intercluster interactions with the La substitution. Also, the values of $E_{a} / k_{B}$ are $\approx 3 T_{0}$ for all $x \leqslant 0.4$ samples (see Table $\mathrm{V}$ ), which is the evidence of the significant coupling between the spin clusters [68]. 

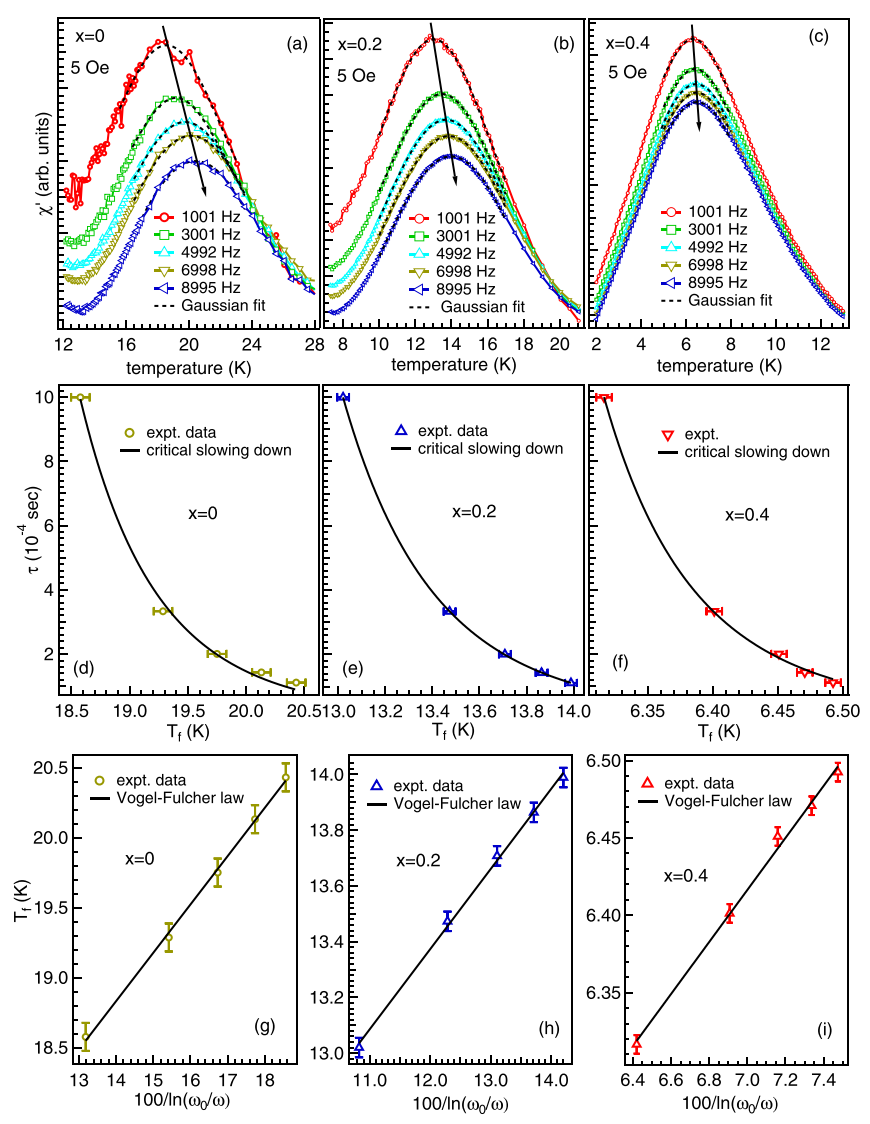

FIG. 11. (a)-(c) The temperature-dependent ac susceptibility curves for the $x=0-0.4$ samples [see the insets of Figs. 10(a), 10 (c) and 10(e), respectively] presented after linear reference line subtraction. The black dotted lines represent the Gaussian fitting of the data points in the vicinity of the freezing temperature $\left(T_{f}\right)$ to estimate the exact value of $T_{f}$. The arrows indicate the shift in $T_{f}$ to the higher temperature with increase in the excitation frequency. (d)-(f) The dependence of spin relaxation time, $\tau$ on the freezing temperature $\left(T_{f}\right)$ along with the best fit (solid black lines) using the critical slowing down model. (g)-(i) The dependence of the freezing temperature on the excitation frequency plotted as $T_{f}$ vs $100 / \ln \left(\omega_{0} / \omega\right)$ along with the best fit (solid black lines) using the Vogel-Fulcher law.

It is important to note that the degree of antisite disorder in these samples decreases with increase in the La concentration, as quantitatively estimated by refining the occupancy of $B$-site cations at their respective Wyckoff positions during the Rietveld refinement of the XRD data data [39]. In the $x=0$ sample, the $\mathrm{Co}^{3+}$ ions are randomly occupied at the $B$ sites, and resulting in the $\mathrm{LS} \mathrm{Co}^{3+}-\mathrm{O}-\mathrm{HS} \mathrm{Co}^{3+}$ and $\mathrm{HS}$ $\mathrm{Co}^{3+}-\mathrm{O}-\mathrm{HS} \mathrm{Co}^{3+}$ interactions, which are expected to be FM and AFM, respectively, as per the Goodenough-Kanamori rules $[78,79]$. These competing interactions result in the spin frustration, which gives rise to the low-temperature glassy behavior in these samples. However, with the La substitution the $\mathrm{Co}^{2+}$ concentration and hence $B$-site ordering increases, which results in the formation of ordered $\mathrm{Co}^{2+}-\mathrm{O}-\mathrm{Nb}^{5+}-\mathrm{O}-$ $\mathrm{Co}^{2+}$ path and suppression of the $\mathrm{Co}^{3+}-\mathrm{O}-\mathrm{Co}^{3+}$ channel and consequently reduction in the FM interactions [39]. In this context, the magnetic entropies due to the Schottky anomaly
TABLE V. The fitting parameters extracted from the frequency dependence of the ac- $\chi$ for the $x=0-0.4$ samples.

\begin{tabular}{llll}
\hline \hline & $x=0$ & $x=0.2$ & $x=0.4$ \\
\hline$\partial T_{f}$ & 0.105 & 0.078 & 0.029 \\
$\tau_{0}(\mathrm{sec})$ & $5.09 \times 10^{-7}$ & $9.73 \times 10^{-8}$ & $1.72 \times 10^{-10}$ \\
$T_{\mathrm{SG}}(\mathrm{K})$ & $13.5(2)$ & $9.7(2)$ & $5.9(1)$ \\
$\mathrm{z} v$ & $7.7(3)$ & $8.4(6)$ & $5.8(1)$ \\
$E_{a} / \mathrm{k}_{\mathrm{B}}(\mathrm{K})$ & $34.6(7)$ & $28.8(7)$ & $16.8(6)$ \\
$T_{0}(\mathrm{~K})$ & $14.0(1)$ & $9.9(1)$ & $5.3(1)$ \\
\hline \hline
\end{tabular}

present in the specific heat curves of $x \leqslant 0.4$ samples are significantly lower than the theoretically expected values, i.e., $R \ln \Omega$ [see Figs. 4(d)-4(f) and discussion therein], indicting the formation of the magnetic clusters at low temperature rather than long-range magnetic ordering. Here, the magnetic entropy associated with each cluster of free spins can be expressed as $S_{\mathrm{cl}}=k_{\mathrm{B}} \ln 2$ [80]. If there are total $N_{\mathrm{cl}}$ clusters with each having $N_{S}$ spins, then the magnetic entropy $\left(S_{\mathrm{mag}}\right)$ per mole of spins can be written as $R \ln 2 / N_{\mathrm{S}}[68,81]$. The values of $S_{\text {mag }}$ for the $x=0,0.2$, and 0.4 samples above their respective freezing temperatures are $0.6,0.9$, and 1.6 J/mole-K, respectively, see Figs. 4(d)-4(f). Using these $S_{\text {mag }}$ values, we estimate the $N_{S} \approx 10,6$, and 4 for the $x=0,0.2$, and 0.4 samples, respectively, which point toward decrease in the typical cluster size with La substitution for the $x \leqslant$ 0.4 samples. In the present case, the $\mathrm{HS}-\mathrm{Co}^{2+}$ shows the long-range AFM exchange interaction with the nearest neighbor $\mathrm{HS}-\mathrm{CO}^{2+}$ in the octahedral coordination [31]. Thus, the suppression of the FM clusters and monotonic enhancement in the AFM coupling with the La substitution are responsible for the observed reduction in the intercluster interaction as well as spin-spin correlation strength within the glassy clusters, as evident from the decrease in the values of $T_{0}$ and $\tau_{0}$ (see Table V). However, considering the localized nature of the spins in the present case, field-dependent small-angle neutron scattering can be used for accurate determination of the cluster size, which is out of the scope of this paper. The presence of glassy behavior due to the interactions between FM and AFM clusters has been well established for the phase-segregated perovskites $[82,83]$. Also, the coexistence of the cluster SG and short- (long-) range AFM exchange interactions has been reported in $\mathrm{Ca}(\mathrm{Pb}) \mathrm{Fe}_{0.5} \mathrm{Nb}_{0.5} \mathrm{O}_{3}$ samples $[84,85]$.

Finally, in Fig. 12, we present the phase diagram of $\mathrm{Sr}_{2-x} \mathrm{La}_{x} \mathrm{CoNbO}_{6}(x=0-1)$ to show the evolution of the different magnetic interactions with temperature, magnetic field, and La concentration $(x)$. Here, the red color indicates the presence of cluster-glass-like behavior for the $x \leqslant 0.4$ samples below $T_{\mathrm{SG}}$ (see Table $\mathrm{V}$ ), whereas $\mathrm{PM}$ behavior at higher temperatures is shown by the green color for all the samples. The magnetic field and temperature dependence of the AFM interactions in the $x \geqslant 0.6$ samples is represented by yellow color. We use the inverse magnetocaloric effect (IMCE) to conventional magnetocaloric effect crossover temperature of the magnetic entropy change $(\Delta S)$ curves as the $T_{N}$ in the case of the $x=0.6$ sample for different applied magnetic fields (see Figs. 5(a)-5(c) of Ref. [61]) due to broad peak in $C_{P}(H, T)$ curves [see inset of Fig. 5(a)]. The continuous 


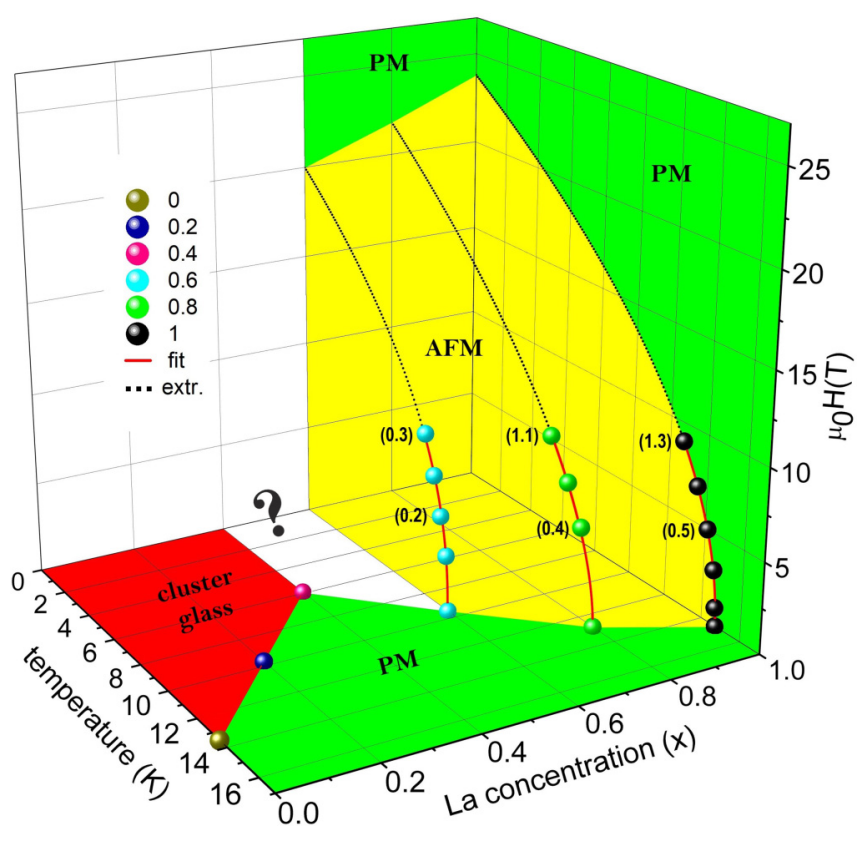

FIG. 12. The phase diagram of $\operatorname{Sr}_{2-x} \mathrm{La}_{x} \mathrm{CoNbO}_{6}(x=0-1)$, where different colors represent the evolution of various magnetic interactions with temperature, magnetic field, and La concentration $(x)$. The $\Delta S_{\max }(\mathrm{J} / \mathrm{kg}-\mathrm{K})$ values in the vicinity of $T_{N}$ are shown in parentheses at $\Delta \mu_{0} H=5$ and 9 T fields for the $x \geqslant 0.6$ samples.

red lines for the $x \geqslant 0.6$ samples indicate the best fit using the AFM decay model as discussed above and dotted lines are their extrapolation to the $0 \mathrm{~K}$ temperature. We obtained $\psi=0.44(2), H_{0}=20.0(4) \mathrm{T}$, and $T_{N} \approx 9.5 \mathrm{~K}$ for the $x=0.6$ sample. Here, we use the linear interpolation between the two conjugative samples for the $T_{\mathrm{SG}}, T_{N}$, and $H_{0}$. A clear enhancement in the critical magnetic field, $H_{0}$ is observed with the La substitution for the $x \geqslant 0.6$ samples, which indicates the strengthening of the AFM coupling. The numbers in the parentheses represent the $\Delta S_{\max }(\mathrm{J} / \mathrm{kg}-\mathrm{K})$ in the vicinity of $T_{N}$ for the $x \geqslant 0.6$ samples at $\Delta \mu_{0} H=5$ and $9 \mathrm{~T}$, see detailed analysis in Figs. 5(a)-5(c) of Ref. [61]. An increase in the value of $\Delta S_{\max }$ with $x$ directly evidencing the enhancement in IMCE due to strengthening of this AFM coupling. It is important to mention that a transition from cluster-glass to AFM ground state has been observed between the $x=0.4$ to 0.6 samples. This motivates further experimental and theoretical investigation of the intermediate concentrations to understand the complex magnetic interactions present in these samples.

\section{CONCLUSIONS}

In conclusion, we study the low-temperature complex magnetic interactions in $\mathrm{Sr}_{2-x} \mathrm{La}_{x} \mathrm{CoNbO}_{6}(x=0-1)$ double perovskites by performing detailed measurements and analysis of temperature and magnetic field dependent specific heat $C_{P}(T)$ and ac susceptibility. We found the high value of Debye temperature from the analysis of $C_{P}(T)$ data for all the samples. Our analysis of the temperature and field-dependent behavior of Schottky anomaly present in the $x \leqslant 0.4$ samples indicate the presence of the spin-orbit triplet of $\mathrm{HS} \mathrm{Co}^{3+}$ just above the LS state and the extracted value of the Landé $g$ factor $(\approx 3)$ discard the presence of the IS state of $\mathrm{Co}^{3+}$ in this series of the samples. The specific-heat data show the evolution of $\lambda$-type anomaly in the $x \geqslant 0.6$ samples and detailed analysis indicates the second-order AFM-PM phase transition and having 3D Heisenberg-like isotropic magnetic interactions below the $T_{N}$. The calculated value of the magnetic entropy and peak jump in $C_{P}(T)$ curves in case of the $x=1$ sample indicate the presence of the free $\mathrm{Co}^{2+}$-like Kramers doublet ground state. We suggest that the persistence of these discrete atomic energy states in these compounds is a valid evidence against the long-standing oversimplified assumption of the complete quenching of the orbital angular moment in $3 d$ complexes, and this will lead to much deeper insight into the complex magnetic interactions and electronic structure of such compounds. Moreover, the ac susceptibility and TRM measurements for the $x \leqslant 0.4$ samples show the low temperature cluster-glass-like behavior owing to the $B$ site disorders, where intercluster interactions and spin-spin interaction strength within the glassy clusters decreases with the La substitution.

\section{ACKNOWLEDGMENTS}

We thank SERB-DST for financial support through an Early Career Research (ECR) Award (Project No. ECR/2015/000159). A.K. thanks UGC for the fellowship. R.S.D. also acknowledges INSA-DFG for Fellowship No. Int/DFG/2019 under the international exchange of scientists program between India and Germany.
[1] A. P. Ramirez, Strongly geometrically frustrated magnets, Annu. Rev. Mater. Sci. 24, 453 (1994).

[2] J. E. Greedan, Geometrically frustrated magnetic materials, J. Mater. Chem. 11, 37 (2001).

[3] S. T. Bramwell and M. J. P. Gingras, Spin ice state in frustrated magnetic pyrochlore materials, Science,294, 1495 (2001).

[4] P. Lampen, N. S. Bingham, M. H. Phan, H. Srikanth, H. T. Yi, and S. W. Cheong, Macroscopic phase diagram and magnetocaloric study of metamagnetic transitions in the spin chain system $\mathrm{Ca}_{3} \mathrm{Co}_{2} \mathrm{O}_{6}$, Phys. Rev. B 89, 144414 (2014).
[5] S. Yamada, N. Abe, H. Sagayama, K. Ogawa, T. Yamagami, and T. Arima, Room-Temperature Low-Field Colossal Magnetoresistance in Double-Perovskite Manganite, Phys. Rev. Lett. 123, 126602 (2019).

[6] J. H. Park, S. K. Kwon, and B. I. Min, Half-metallic antiferromagnetic double perovskites: $\mathrm{LaAVRuO}_{6}(\mathrm{~A}=\mathrm{Ca}, \mathrm{Sr}$, and $\mathrm{Ba})$, Phys. Rev. B 65, 174401 (2002).

[7] J.-W. G. Bos and J. P. Attfield, Magnetic frustration in $(\mathrm{LaA}) \mathrm{CoNbO}_{6}(\mathrm{~A}=\mathrm{Ca}, \mathrm{Sr}$, and $\mathrm{Ba})$ double perovskites, Phys. Rev. B 70, 174434 (2004). 
[8] P. A. Kumar, R. Mathieu, R. Vijayaraghavan, S. Majumdar, O. Karis, P. Nordblad, B. Sanyal, O. Eriksson, and D. D. Sarma, Ferrimagnetism, antiferromagnetism, and magnetic frustration in $\mathrm{La}_{2-x} \mathrm{Sr}_{x} \mathrm{CuRuO}_{6}(0 \leqslant \mathrm{x} \leqslant 1)$, Phys. Rev. B 86, 094421 (2012).

[9] T. Aharen, J. E. Greedan, F. Ning, T. Imai, V. Michaelis, S. Kroeker, H. Zhou, C. R. Wiebe, and L. M. D. Cranswick, Magnetic properties of the $\mathrm{S}=3 / 2$ geometrically frustrated double perovskites $\mathrm{La}_{2} \mathrm{LiRuO}_{6}$ and $\mathrm{Ba}_{2} \mathrm{YRuO}_{6}$, Phys. Rev. B 80, 134423 (2009).

[10] C. R. Wiebe, J. E. Greedan, P. P. Kyriakou, G. M. Luke, J. S. Gardner, A. Fukaya, I. M. Gat-Malureanu, P. L. Russo, A. T. Savici, and Y. J. Uemura, Frustration-driven spin freezing in the $\mathrm{S}=1 / 2$ fcc perovskite $\mathrm{Sr}_{2} \mathrm{MgReO}_{6}$, Phys. Rev. B 68, 134410 (2003)

[11] G. King and P. M. Woodward, Cation ordering in perovskites, J. Mater. Chem. 20, 5785 (2010).

[12] S. Vasala and M. Karppinen, $\mathrm{A}_{2} \mathrm{~B}^{\prime} \mathrm{B}^{\prime \prime} \mathrm{O}_{6}$ perovskites: A review, Prog. Solid State Chem. 43, 1 (2015).

[13] M. J. R. Hoch, P. L. Kuhns, W. G. Moulton, A. P. Reyes, J. Wu, and C. Leighton, Spin dynamics in $\mathrm{La}_{1-x} \mathrm{Sr}_{x} \mathrm{CoO}_{3}$, Phys. Rev. B 69, 014425 (2004).

[14] N. Narayanan, D. Mikhailova, A. Senyshyn, D. M. Trots, R. Laskowski, P. Blaha, K. Schwarz, H. Fuess, and H. Ehrenberg, Temperature and composition dependence of crystal structures and magnetic and electronic properties of the double perovskites $\mathrm{La}_{2-x} \mathrm{Sr}_{x} \mathrm{CoIrO}_{6}(0 \leqslant x \leqslant 2)$, Phys. Rev. B 82, 024403 (2010).

[15] R. Prakash, R. Shukla, P. Nehla, A. Dhaka, and R. S. Dhaka, Tuning ferromagnetism and spin state in $\mathrm{La}_{(1-x)} \mathrm{A}_{x} \mathrm{CoO}_{3}$ $(\mathrm{A}=\mathrm{Sr}, \mathrm{Ca})$ nanoparticles, J. Alloys Compd. 764, 379 (2018)

[16] P. M. Raccah and J. B. Goodenough, First-order localizedelectron $\rightleftarrows$ collective-electron transition in $\mathrm{LaCoO}_{3}$, Phys. Rev. 155, 932 (1967).

[17] V. G. Bhide, D. S. Rajori, G. R. Rao, and C. N. R. Rao, Mössbauer studies of the high-spin-low-spin equilibria and the localized-collective electron transition in $\mathrm{LaCoO}_{3}$, Phys. Rev. B 6, 1021 (1972).

[18] K. Asai, P. Gehring, H. Chou, and G. Shirane, Temperatureinduced magnetism in $\mathrm{LaCoO}_{3}$, Phys. Rev. B 40, 10982 (1989)

[19] R. Mahendiran and A. K. Raychaudhuri, Magnetoresistance of the spin-state-transition compound $\mathrm{La}_{1-x} \mathrm{Sr}_{x} \mathrm{CoO}_{3}$, Phys. Rev. B 54, 16044 (1996).

[20] J. Wu and C. Leighton, Glassy ferromagnetism and magnetic phase separation in $\mathrm{La}_{1-x} \mathrm{Sr}_{x} \mathrm{CoO}_{3}$, Phys. Rev. B 67, 174408 (2003)

[21] R. Shukla and R. S. Dhaka, Anomalous magnetic and spin glass behavior in Nb-substituted $\mathrm{LaCo}_{1-x} \mathrm{Nb}_{x} \mathrm{O}_{3}$, Phys. Rev. B 97, 024430 (2018).

[22] R. Shukla, A. Jain, M. Miryala, M. Murakami, K. Ueno, S. M. Yusuf, and R. S. Dhaka, Spin dynamics and unconventional magnetism in insulating $\mathrm{La}_{(1-2 x)} \mathrm{Sr}_{2 x} \mathrm{Co}_{(1-x)} \mathrm{Nb}_{x} \mathrm{O}_{3}$, J. Phys. Chem. C 123, 22457 (2019).

[23] M. Zhuang, W. Zhang, and N. Ming, Competition of various spin states of $\mathrm{LaCoO}_{3}$, Rev. B 57, 10705 (1998).

[24] M. A. Korotin, S. Y. Ezhov, I. V. Solovyev, V. I. Anisimov, D. I. Khomskii, and G. A. Sawatzky, Intermediate-spin state and properties of $\mathrm{LaCoO}_{3}$, Phys. Rev. B 54, 5309 (1996).

[25] S. Noguchi, S. Kawamata, K. Okuda, H. Nojiri, and M. Motokawa, Evidence for the excited triplet of $\mathrm{Co}^{3+}$ in $\mathrm{LaCoO}_{3}$, Phys. Rev. B 66, 094404 (2002).

[26] Z. Ropka and R. J. Radwanski, ${ }^{5} \mathrm{D}$ term origin of the excited triplet in $\mathrm{LaCoO}_{3}$, Phys. Rev. B 67, 172401 (2003).

[27] C. He, H. Zheng, J. F. Mitchell, M. L. Foo, R. J. Cava, and C. Leighton, Low temperature Schottky anomalies in the specific heat of $\mathrm{LaCoO}_{3}$ : Defect-stabilized finite spin states, Appl. Phys. Lett. 94, 102514 (2009).

[28] A. Podlesnyak, S. Streule, J. Mesot, M. Medarde, E. Pomjakushina, K. Conder, A. Tanaka, M. W. Haverkort, and D. I. Khomskii, Spin-State Transition in $\mathrm{LaCoO}_{3}$ : Direct Neutron Spectroscopic Evidence of Excited Magnetic States, Phys. Rev. Lett. 97, 247208 (2006).

[29] Magnetism and Transition Metal Complexes, edited by F. E. Mabbs and D. J. Machin (Chapman and Hall, London, 1973).

[30] M. C. Viola, M. J. Martínez-Lope, J. A. Alonso, J. L. Martínez, J. M. D. Paoli, S. Pagola, J. C. Pedregosa, M. T. Fernández-Díaz, and R. E. Carbonio, Structure and magnetic properties of $\mathrm{Sr}_{2} \mathrm{CoWO}_{6}$ : An ordered double perovskite containing $\mathrm{Co}^{2+}(\mathrm{HS})$ with unquenched orbital magnetic moment, Chem. Mater. 15, 1655 (2003).

[31] F. Lloret, M. Julve, J. Cano, R. Ruiz-García, and E. Pardo, Magnetic properties of six-coordinated high-spin $\mathrm{Co}(\mathrm{II})$ complexes: Theoretical background and its application, Inorg. Chem. Acta 361, 3432 (2008).

[32] R. P. Madhogaria, R. Das, E. M. Clements, V. Kalappattil, M. H. Phan, H. Srikanth, N. T. Dang, D. P. Kozlenko, and N. S. Bingham, Evidence of long-range ferromagnetic order and spin frustration effects in the double perovskite $\mathrm{La}_{2} \mathrm{CoMnO}_{6}$, Phys. Rev. B 99, 104436 (2019).

[33] R. C. Sahoo, Y. Takeuchi, A. Ohtomo, and Z. Hossain, Exchange bias and spin glass states driven by antisite disorder in the double perovskite compound $\mathrm{LaSrCoFeO}_{6}$, Phys. Rev. B 100, 214436 (2019).

[34] G. R. Haripriya, C. M. N. Kumar, R. Pradheesh, L. M. Martinez, C. L. Saiz, S. R. Singamaneni, T. Chatterji, V. Sankaranarayanan, K. Sethupathi, B. Kiefer, and H. S. Nair, Contrasting the magnetism in $\mathrm{La}_{2-x} \mathrm{Sr}_{x} \mathrm{FeCoO}_{6}(x=0,1,2)$ double perovskites: The role of electronic and cationic disorder, Phys. Rev. B 99, 184411 (2019).

[35] R. I. Dass and J. B. Goodenough, Multiple magnetic phases of $\mathrm{La}_{2} \mathrm{CoMnO}_{6-\delta}(0 \leqslant \delta \leqslant 0.05)$, Phys. Rev. B 67, 014401 (2003).

[36] A. N. Vasiliev, O. S. Volkova, L. S. Lobanovskii, I. O. Troyanchuk, Z. Hu, L. H. Tjeng, D. I. Khomskii, H.-J. Lin, C. T. Chen, N. Tristan, F. Kretzschmar, R. Klingeler, and B. Büchner, Valence states and metamagnetic phase transition in partially B-site-disordered perovskite $\mathrm{EuMn}_{0.5} \mathrm{Co}_{0.5} \mathrm{O}_{3}$, Phys. Rev. B 77, 104442 (2008).

[37] R. D. Shannon, Revised effective ionic radii and systematic studies of interatomic distances in halides and chalcogenides, Acta. Cryst. A 32, 751 (1976).

[38] M. T. Azcondo, J. R. de Paz, K. Boulahya, C. Ritter, F. García-Alvarado, and U. Amador, Complex magnetic behavior of $\mathrm{Sr}_{2} \mathrm{CoNb}_{1-x} \mathrm{Ti}_{x} \mathrm{O}_{6}(0 \leqslant x \leqslant 0.5)$ as a result of a flexible microstructure, Dalton Trans. 44, 3801 (2015). 
[39] A. Kumar and R. S. Dhaka, Unraveling magnetic interactions and the spin state in insulating $\mathrm{Sr}_{2-x} \mathrm{La}_{x} \mathrm{CoNbO}_{6}$, Phys. Rev. B 101, 094434 (2020).

[40] A. Kumar, R. Shukla, A. Pandey, S. Dalal, M. Miryala, K. Ueno, M. Murakami, and R. S. Dhaka, Structural, transport, optical, and electronic properties of $\mathrm{Sr}_{2} \mathrm{CoNbO}_{6}$ thin films, J. Appl. Phys. 128, 025303 (2020).

[41] C. Kittel, Introduction to Solid State Physics, 8th ed. (Wiley, New York, 2005).

[42] H. Liu and G. Khaliullin, Pseudospin exchange interactions in $\mathrm{d}^{7}$ cobalt compounds: Possible realization of the Kitaev model, Phys. Rev. B 97, 014407 (2018).

[43] A. Tari, The Specific Heat of Matter at Low Temperature (Imperial College Press, London, 2003).

[44] Y. Hinatsu and Y. Doi, Magnetic susceptibility and specific heat of uranium double perovskite oxides $\mathrm{Ba}_{2} \mathrm{MUO}_{6}(\mathrm{M}=\mathrm{Co}, \mathrm{Ni})$, J. Solid State Chem. 179, 2079 (2006).

[45] S. R. Gopal, Specific Heats at Low Temperatures (Plenum, New York, 1966).

[46] R. J. Goetsch, V. K. Anand, A. Pandey, and D. C. Johnston, Structural, thermal, magnetic, and electronic transport properties of the $\mathrm{LaNi}_{2}\left(\mathrm{Ge}_{1-x} \mathrm{P}_{x}\right)_{2}$ system, Phys. Rev. B 85, 054517 (2012).

[47] C. He, S. Eisenberg, C. Jan, H. Zheng, J. F. Mitchell, and C. Leighton, Heat capacity study of magnetoelectronic phase separation in $\mathrm{La}_{1-x} \mathrm{Sr}_{x} \mathrm{CoO}_{3}$ single crystals, Phys. Rev. B 80, 214411 (2009).

[48] M. Mattesini, M. Magnuson, F. Tasnádi, C. Höglund, I. A. Abrikosov, and L. Hultman, Elastic properties and electrostructural correlations in ternary scandium-based cubic inverse perovskites: A first-principles study, Phys. Rev. B 79, 125122 (2009).

[49] L. T. Corredor, G. Aslan-Cansever, M. Sturza, K. Manna, A. Maljuk, S. Gass, T. Dey, A. U. B. Wolter, O. Kataeva, A. Zimmermann, M. Geyer, C. G. F. Blum, S. Wurmehl, and B. Büchner, Iridium double perovskite $\mathrm{Sr}_{2} \mathrm{YIrO}_{6}$ : A combined structural and specific heat study, Phys. Rev. B 95, 064418 (2017).

[50] M. Watanabe, N. Kurita, H. Tanaka, W. Ueno, K. Matsui, and T. Goto, Valence-bond-glass state with a singlet gap in the spin-1/2 square-lattice random $\mathrm{J}_{1}-\mathrm{J}_{2}$ Heisenberg antiferromagnet $\mathrm{Sr}_{2} \mathrm{CuTe}_{1-x} \mathrm{~W}_{x} \mathrm{O}_{6}$, Phys. Rev. B 98, 054422 (2018).

[51] B. Bleaney, Nuclear specific heats in paramagnetic salts, Phys. Rev. 78, 214 (1950).

[52] J. E. Gordon, R. A. Fisher, Y. X. Jia, and N. E. Phillips, S. F. Reklis, D. A. Wright, and A. Zettl, Specific heat of $\mathrm{Nd}_{0.67} \mathrm{Sr}_{0.33} \mathrm{MnO}_{3}$, Phys. Rev. B 59, 127 (1999).

[53] Y. S. Orlov, V. A. Dudnikov, M. S. Platunov, M. V. Gorev, D. A. Velikanov, N. V. Kazak, S. Y. Gavrilkin, L. A. Solov'ev, A. A. Veligzhanin, S. N. Vereshchagin, and S. G. Ovchinnikov, Lowtemperature Schottky anomalies and the magnetic state of the $\mathrm{p}$ electrons of oxygen in substituted $\mathrm{Gd}_{0.4} \mathrm{Sr}_{0.6} \mathrm{CoO}_{3-\delta}$ cobaltites, J. Exp. Theor. Phys. 126, 217 (2018).

[54] A. Deb, J. M. Ralph, E. J. Cairns, and U. Bergmann, Characterization of $\mathrm{La}_{0.8} \mathrm{Sr}_{0.2} \mathrm{FeO}_{3-\delta}$ and $\mathrm{La}_{0.7} \mathrm{Sr}_{0.2} \mathrm{FeO}_{3-\delta}$ as a function of temperature by x-ray absorption spectroscopy, Phys. Rev. B 73, 115114 (2006).

[55] A. Szewczyk, M. Gutowska, and B. Dabrowski, Specific heat and phase diagram of heavily doped $\mathrm{La}_{1-x} \mathrm{Sr}_{x} \mathrm{MnO}_{3}(0.45 \leqslant$ $x \leqslant 1.0$ ), Phys. Rev. B 72, 224429 (2005).
[56] L. J. de Jongh and A. R. Miedema, Experiments on simple magnetic model systems, Adv. Phys. 23, 1 (1974).

[57] M. Marinelli, F. Mercuri, U. Zammit, R. Pizzoferrato, F. Scudieri, and D. Dadarlat, Photopyroelectric study of specific heat, thermal conductivity, and thermal diffusivity of $\mathrm{Cr}_{2} \mathrm{O}_{3}$ at the Néel transition, Phys. Rev. B 49, 9523 (1994).

[58] A. Oleaga, A. Salazar, D. Prabhakaran, J.-G. Cheng, and J.-S. Zhou, Critical behavior of the paramagnetic to antiferromagnetic transition in orthorhombic and hexagonal phases of $\mathrm{RMnO}_{3}(\mathrm{R}=\mathrm{Sm}, \mathrm{Tb}, \mathrm{Dy}, \mathrm{Ho}, \mathrm{Er}, \mathrm{Tm}, \mathrm{Yb}, \mathrm{Lu}, \mathrm{Y})$, Phys. Rev. B 85, 184425 (2012).

[59] G. Ahlers, Critical phenomena at low temperature, Rev. Mod. Phys 52, 489 (1980).

[60] J. C. Le Guillou and J. Zinn-Justin, Critical exponents from field theory, Phys. Rev. B 21, 3976 (1980).

[61] See Supplemental Material at http://link.aps.org/supplemental/ 10.1103/PhysRevB.102.184414 for further information about thermoremanent magnetization and aging data as well as the analysis of the magnetic entropy and adiabatic temperature change, which also includes Refs. [62-67].

[62] K. Mukherjee and A. Banerjee, Changeover from glassy ferromagnetism of the orbital domain state to long-range ferromagnetic ordering in $\mathrm{La}_{0.9} \mathrm{Sr}_{0.1} \mathrm{MnO}_{3}$, Phys. Rev. B 77, 024430 (2008).

[63] A. Midya, P. Mandal, K. Rubi, R. Chen, J. S. Wang, R. Mahendiran, G. Lorusso, and M. Evangelisti, Large adiabatic temperature and magnetic entropy changes in $\mathrm{EuTiO}_{3}$, Phys. Rev. B 93, 094422 (2016).

[64] M. H. Phan and S. C. Yu, Review of magnetocloric effect in manganite materials, J. Magn. Magn. Mater. 308, 325 (2007).

[65] V. K. Pecharsky and K. A. Gschneidner, Jr., Magnetocaloric effect from indirect measurements: Magnetization and heat capacity, J. Appl. Phys. 86, 565 (1999).

[66] T. Krenke, E. duman, M. Acet, E. F. Wassermann, X. Moya, L. Mañosa, and A. Planes, Inverse magnetocaloric effect in ferromagnetic Ni-Mn-Sn alloys, Nature Mater 4, 450 (2005).

[67] Proceedings of the First International Conference on Magnetic Refrigeration at Room Temperature, edited by P. W. Egolf, (International Institute of Refrigeration, Paris, France, 2005).

[68] V. K. Anand, D. T. Adroja, and A. D. Hillier, Ferromagnetic cluster spin-glass behavior in $\operatorname{PrRhSn}_{3}$, Phys. Rev. B 85, 014418 (2012).

[69] V. Markovich, I. Fita, A. Wisniewski, G. Jung, D. Mogilyansky, R. Puzniak, L. Titelman, and G. Gorodetsky, Spin-glass-like properties of $\mathrm{La}_{0.8} \mathrm{Ca}_{0.2} \mathrm{MnO}_{3}$ nanoparticles ensembles, Phys. Rev. B 81, 134440 (2010).

[70] K. Binder and A. P. Young, Spin glasses: Experimental facts, theoretical concepts, and open questions, Rev. Mod. Phys. 58, 801 (1986).

[71] J. A. Mydosh, Spin Glasses: An Experimental Introduction, Vol. 125 (Taylor and Francis, London, 1993).

[72] M. Giot, A. Pautrat, G. André, D. Saurel, M. Hervieu, and J. Rodriguez-Carvajal, Magnetic states and spin-glass properties in $\mathrm{Bi}_{0.67} \mathrm{Ca}_{0.33} \mathrm{MnO}_{3}$ : Macroscopic ac measurements and neutron scattering, Phys. Rev. B 77, 134445 (2008).

[73] P. C. Hohenberg and B. I. Halperin, Theory of dynamic critical phenomena, Rev. Mod. Phys. 49, 435 (1977).

[74] J. Souletie and J. L. Tholence, Critical slowing down in spin glasses and other glasses: Fulcher versus power law, Phys. Rev. B 32, 516 (1985). 
[75] A. T. Ogielski and I. Morgenstern, Critical Behavior of ThreeDimensional Ising Spin-Glass Model, Phys. Rev. Lett. 54, 928 (1985).

[76] J. Lago, S. J. Blundell, A. Eguia, M. Jansen, and T. Rojo, Three-dimensional Heisenberg spin-glass behavior in $\mathrm{SrFe}_{0.90} \mathrm{Co}_{0.10} \mathrm{O}_{3.0}$, Phys. Rev. B 86, 064412 (2012).

[77] A. Malinowski, V. L. Bezusyy, R. Minikayev, P. Dziawa, Y. Syryanyy, and M. Sawicki, Spin-glass behavior in Ni-doped $\mathrm{La}_{1.85} \mathrm{Sr}_{0.15} \mathrm{CuO}_{4}$, Phys. Rev. B 84, 024409 (2011).

[78] J. B. Goodenough, Theory of the role of covalence in the perovskite-type manganites [La, $\mathrm{M}(\mathrm{II})] \mathrm{MnO}_{3}$, Phys. Rev. 100, 564 (1955).

[79] J. Kanamori, Superexchange interactions and symmetry properties of electron orbitals, J. Phys. Chem. Solids 10, 87 (1959).

[80] M. Cyrot, Clusters and the spin-glass transition, Solid State Commun. 39, 1009 (1981).
[81] E. Miranda and V. Dobrosavljević, Disorder-driven non-Fermi liquid behavior of correlated electrons, Rep. Prog. Phys. 68, 2337 (2005).

[82] Y. K. Tang, Y. Sun, and Z. H. Cheng, Glassy magnetic behavior in the phase-separated perovskite cobaltites, Phys. Rev. B 73, 012409 (2006).

[83] F. Rivadulla, M. A. L.- Quintela, and J. Rivas, Origin of the Glassy Magnetic Behavior of the Phase Segregated State of the Perovskites, Phys. Rev. Lett. 93, 167206 (2004).

[84] A. Kumar, A. Senyshyn, and D. Pandey, Evidence for cluster spin glass phase with precursor short-range antiferromagnetic correlations in the B-site disordered $\mathrm{Ca}\left(\mathrm{Fe}_{1 / 2} \mathrm{Nb}_{1 / 2}\right) \mathrm{O}_{3}$ perovskite, Phys. Rev. B 99, 214425 (2019).

[85] W. Kleemann, V. V. Shvartsman, P. Borisov, and A. Kania, Coexistence of Antiferromagnetic and Spin Cluster Glass Order in the Magnetoelectric Relaxor Multiferroic $\mathrm{PbFe}_{0.5} \mathrm{Nb}_{0.5} \mathrm{O}_{3}$, Phys. Rev. Lett. 105, 257202 (2010). 